\title{
Design of a 4-DOF grounded exoskeletal robot for shoulder and elbow rehabilitation
}

\author{
Fatih Karadeniz ${ }^{1}$, Özgür Ege Aydoğan ${ }^{1}$, Emin Abdullah Kazancı ${ }^{1}$, Erhan Akdoğan ${ }^{*}$ \\ ${ }^{1}$ Department of Mechatronics Engineering, Y1ldız Technical University, Istanbul, Turkey
}

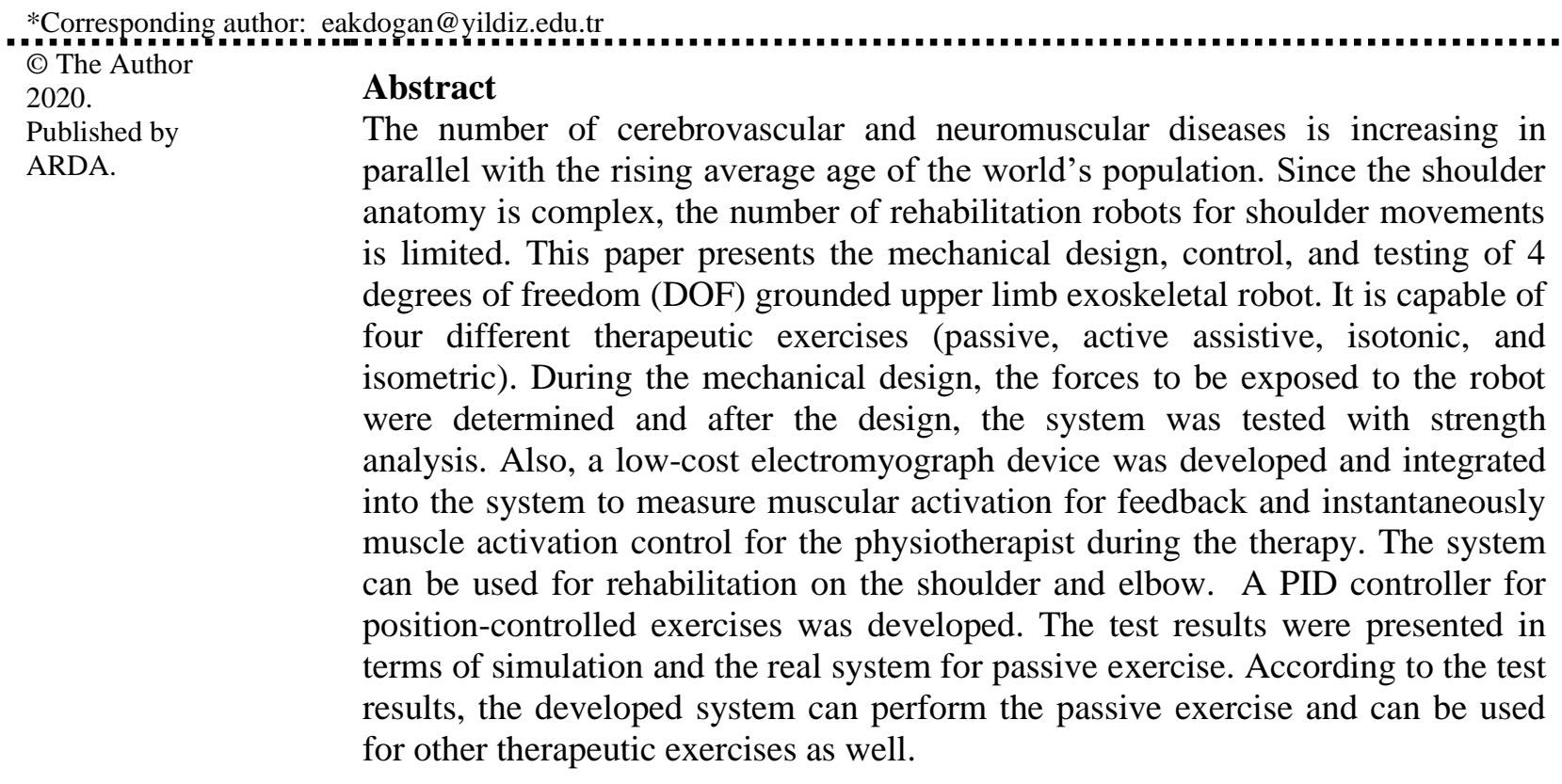

Keywords: Rehabilitation robot, Exoskeletal, Biomechatronics, EMG

(electromyography)

\section{Introduction}

Rehabilitation is a general treatment process that covers all the necessary stages that enable people who have lost some of their physical abilities or limbs as a result of an illness or accident, to re-adapt to life, to improve their life quality and to live seamlessly with their families and society [1].

The number of cerebrovascular and neuromuscular diseases is increasing in parallel with the rising average age of the world's population. For example, as reported by the Council for Economic Planning and Development in Taiwan [2], the percentage of the aged community is predicted to reach $39.4 \%$ in 2060 in Taiwan. Because of that, usage of the rehabilitation robots for physiotherapy of patients who have lost their limb motor functions gains importance.

The World Health Organization's (WHO) estimation regarding the number of individuals which would be affected by chronic diseases by 2030 is \% 23.3 [3]. The number of rehabilitation robots developed especially after the 2000s is increasing day by day. This growth continues steadily. Since the shoulder anatomy is complex, the number of rehabilitation robots for shoulder movements is limited. In this study, a grounded type rehabilitation robot was developed. The literature has been researched in accordance with this type of robot. One of the world-wide studies in this field is the robot MIME [4]. This robot has 6 degrees of freedom and is used for wrist and shoulder rehabilitation. With the help of a Programmable Universal Manipulation Arm

This work is licensed under a Creative Commons Attribution License (https://creativecommons.org/licenses/by/4.0/ ) that allows others to share and adapt the material for any purpose (even commercially), in any medium with an acknowledgement of the work's authorship and initial publication in this journal. 
(PUMA), the system applies the same movements as the intact arm to the rehabilitated. A 5 DOF haptic arm is designed for training and rehabilitation by Gupta and O'Malley [5]. The system works with kinesthetic feedback equipped for the joints of the lower arm and wrist of the operator. Vertechy et al. [6] introduced an upper limb exoskeleton design with a modular custom-designed architecture for upper limb rehabilitation. The system meets the requirements for patient safety and high-performance application flexibility. Ren et al [7] designed a user-friendly robot with single motor named IntelliArm which is able to controlling the whole arm consisting hand opening and closing mechanism separately and synchronically by using $8+2 \mathrm{DOF}$. Ganesan et al. [8] used EMG and IMU in the upper limb exoskeleton design. The system uses the data from the good hand as a feedback and controls the rehabilitation process. Lenzi et al. [9] worked on 10 healthy person's proportional EMG reactions applied by an elbow driven exoskeleton. There are many other examples of upper limb rehabilitation [10 - 11].

Some of the rehabilitation robots for upper limb which are grounded and works on the shoulder region; Hsieh et al. [12] has produced a parallel actuated shoulder mechanism which has two spherical mechanisms, two slider crank mechanisms and a gravity balancing mechanism. As a conclusion of the design, the robot has superior inertia properties and is compact and light. Also, it has an adaptive mechanism that can resolve the misalignment problems. Hunt et al. [13] introduced a five degree of freedom shoulder exoskeleton. One of the main properties of the system is low inertia. The system consists of three parallel linear actuators coupled to the shoulder joint with a three DOF tie-rod joint. The shoulder joint and upper arm are connected to each other with the help of a passive sliding interface. Oguntosin et al. [14] demonstrated an exoskeleton actuated with the soft modules which consists 3D-printed parts. With the light weight of 3D parts, the aim was to compensate gravity and with active joints to rotate the shoulder and elbow joints. The system contains soft materials and pneumatic actuation systems. Shao et al. [15] designed a three DOF cable-driven upper-arm exoskeleton. For an optimal design two adequate systems are proposed according to the analysis. Wu et al. [16] developed a gravity balanced rehabilitation robot for upper limb. The kinematic structure is obtained in a way which interaction is available. As a hybrid combination, auxiliary links are used to balance the effect of gravity with zero-free length springs. Accogli et al. [17] provided an exoskeleton with a cooperative humanmachine interface. Also, EMG based advanced machine learning algorithms are used for motion detection and decoding movement direction. Xu et al. [18] made a research about the muscle condition during and after the rehabilitation session. This study focused especially on muscle fatigue. Riani et al. [19] used adaptive integral terminal sliding mode control for passive exercises. This approach is used for uncertainties of a nonlinear system. Zhang et al. [20] made a study for the complex structure of the glenohumeral joint in the shoulder. A compatible exoskeleton which has 11 degrees of freedom is designed in this study. Alawan et al. [21] worked on induction motor simulations. The PI control of the motors are presented with Neural Network for IM (Induction Motor) speed control. Atiyah et al. [22] made a laboratory study about real time PID control. PLC application was used with liquid level and flow control experiments. Zhang et al. [23] reviewed the designs, sensing and control system designs for knee rehabilitation robots. A detailed approach was made in this study. Zhao et al. [24] proposed a design for an upper limb rehabilitation robot. The system consists of environmentally intelligent ergonomics with multi - channel information interaction technology and big data analysis.

Gopura et al. [25] made a research about existing robots, their problems and history. According to this study they discussed the future of upper limb exoskeletons. Rodic et al. [26] also made a study on the exoskeleton design in the 1960's and 1970's. A comparison was made between today's studies and the previous ones. Considering all these studies, existing grounded upper limb exoskeleton rehabilitation robots have some problems which are listed below;

- The glenohumeral joint in the shoulder is inside the body. Because of that, it is very hard to set the rotation axis of the robot and rotation axis parallel.

- When the robots in the market are analysed, it can be seen that a lot of the robots are bulky and big.

- Some robots have a solid structure which is not adjustable. Because of that patient with different body sizes have difficulties.

In this study, design, producing and control of a 4 DOF grounded upper limb exoskeletal rehabilitation robot for the shoulder area which can be used by elderly, stroke or Parkinson patients was performed. The mechanical design will include three active shoulder movements and one passive elbow movement. These 
movements are; shoulder vertical flexion-extension, shoulder horizontal flexion-extension, shoulder abduction/adduction and elbow flexion-extension. The robot will be able to do these exercises in passive, active-assistive, isotonic and isometric modes. The mechanical design, dynamic model and test results in terms of simulations and real time results for the passive exercises are presented.

\section{Theory of shoulder rehabilitation}

\subsection{Movements}

The dominant joint which connects upper limb to trunk is the glenohumeral joint (shoulder joint). Rehabilitation robot is able to perform vertical flexion-extension, horizontal flexion-extension and abductionadduction for shoulder which can be seen in Fig.1 [27]. Also, vertical flexion-extension for elbow. The definitions of these movements are explained below.
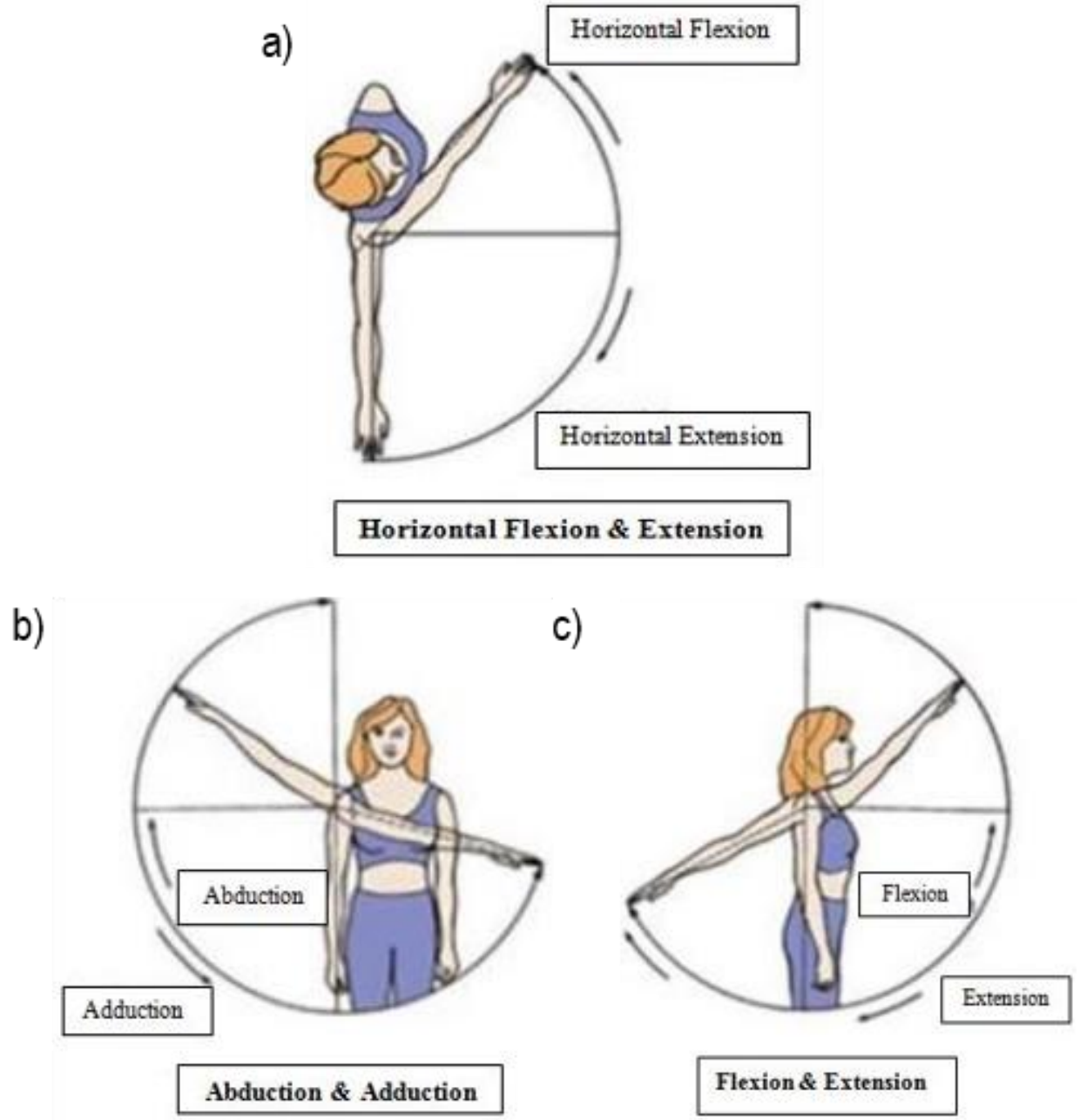

Figure 1. Shoulder exercises: a) horizontal abduction-adduction, b) vertical flexion-extension, c) horizontal flexion-extension [27]

Horizontal flexion - extension: lifting the arm is called extension and lowering the flexion movement. The arm is straight and parallel to ground at start and perpendicular to ground at the end or vice versa.

Vertical flexion - extension: lifting the arm is called extension and lowering the flexion movement. The arm is straight and parallel to ground at start and perpendicular to ground at the end or vice versa.

Abduction-adduction: lifting the arm is called abduction and lowering the adduction movement. The arm is straight and parallel to the body at start and perpendicular to ground at the end or vice versa.

The agonist and antagonist muscles used for each movement are represented on the Tab.1. Antagonist is the name of the straitening muscle and antagonist is the expanding muscle. The surface electrodes are placed on these muscle pairs to measure the muscle activation level by EMG circuit. 
Table 1. Movement - muscle groups

\begin{tabular}{ccc}
\hline Movement & Agonist Muscle & Antagonist Muscle \\
\hline Shoulder Flexion & Anterior Deltoid & Posterior Deltoid \\
Shoulder Extension & Posterior Deltoid & Anterior Deltoid \\
Shoulder Abduction & Medial Deltoid & Latissimus Dorsi \\
Shoulder Adduction & Latissimus Dorsi & Medial Deltoid \\
Elbow Flexion & Biceps & Triceps \\
Elbow Extension & Triceps & Biceps \\
\hline
\end{tabular}

\subsection{Therapeutic exercises}

The rehabilitation robot developed in this study can perform passive, active-assistive, isometric and isotonic exercises for shoulder rehabilitation. The definitions of these are given below.

Passive exercise: The robot moves the extremity of the patient all by itself.

Active - assistive exercise: The patient tries to make the movement and the robot will help the patient when he/she needs.

Isometric exercise: The length of the muscle stays unchanged while muscle activity rises.

Isotonic exercise: The limb works against a constant force which can be changed according to improvements and needs of the patient.

\section{Materials and methods}

\subsection{Functional requirements and design parameters}

The Functional requirements of the developed exoskeletal robot are as follows:

- Capable of shoulder and elbow movement.

- The length should be adjustable related to limb length.

- To protect the patient against problems, the system should be protected with mechanical, electrical and software precautions.

- The robot should be able to make passive, active, isometric and isotonic exercises.

- It should be user friendly.

The design parameters that meet the functional requirements are as follows:

- A robot manipulator with 4 DOF should be designed.

- A mechanism capable of adjusting the length depending on the limb length should be designed.

- Mechanical safety with mechanical limits, electrical safety with emergency stop button and software safety with emergency code has to be provided for the system.

- The motors, reducers, encoders, force sensors and EMGs should be used in the system to actuation and feedback. Force and position control algorithm should be developed to perform related exercises.

- A graphical user interface that the user can use comfortably should be designed.

\subsection{Mechanical design and structure}

The mechanical design was made by looking at the design parameters. The robot is developed for home-based robotic rehabilitation for disabled and aged patients in any circumstances. Therefore, the system must be simple, easy to transfer and can be used by any patient without much effort for adjustments. According to these needs it is designed basically and can be moved to anywhere needed on a platform. Also, it has an adjustable mechanism which can be changed easily. 
The system is designed for patients whose heights are between $1.5-2 \mathrm{~m}$ and weights are maximum $100 \mathrm{~kg}$. According to studies from Rosales et al. [28] the arm weight of a $100 \mathrm{~kg}$ person is $2.6 \mathrm{~kg}$; the forearm weight is $1,6 \mathrm{~kg}$ and the hand weight is $0,7 \mathrm{~kg}$. Also, the length of arm is between 1,5-2 meters. The ranges for the movements are given in Tab.2. In the design the ranges are considered and the robot is designed in a way which it will work in the ranges. If a failure happens and the robot is going out of range, the mechanical precautions are there for stop the system. The range limitations are very important for safety. For smooth movement, bearings are used on the rotating parts according to calculations. Because of weight, cost and rust protection 6013 Aluminium material is used. 6013 Aluminium has a density of $2.72 \mathrm{~g} / \mathrm{cm}^{3}$ and $393 \mathrm{MPa}$ yield strength. Besides, aluminium is one of the most suitable materials that can be used in the robot since it is a light and durable material. Feeders and other enhancer parts are used to make the system reliable and stronger. The last form of the design and produced prototype can be seen in Fig. 2.

Table 2. Movement ranges

\begin{tabular}{cc}
\hline Motion & Range \\
\hline Shoulder (horizontal) flexion (Flex.) / ex-tension (Ext.) & $135^{\circ} / 50^{\circ}$ \\
Shoulder adduction (Add.) / abduction (Abd.) & $108^{\circ} / 0^{\circ}$ \\
Shoulder (vertical) flexion (Flex.) / extension (Ext.) & $135^{\circ} / 50^{\circ}$ \\
\hline
\end{tabular}

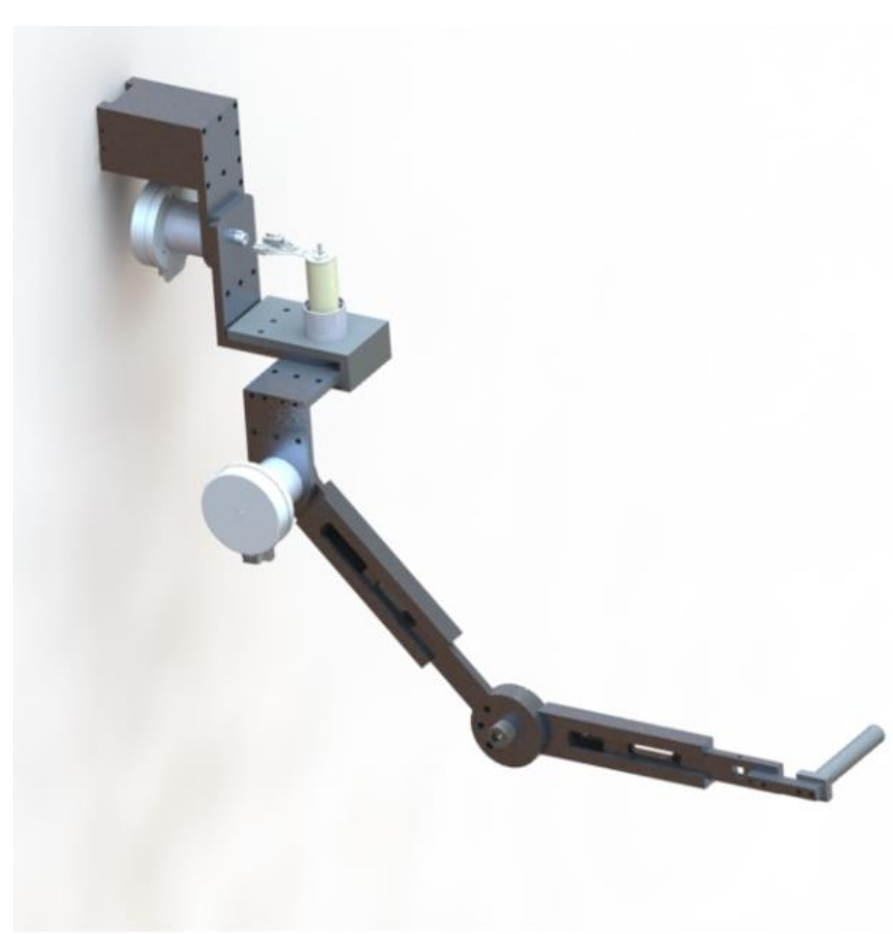

(a)

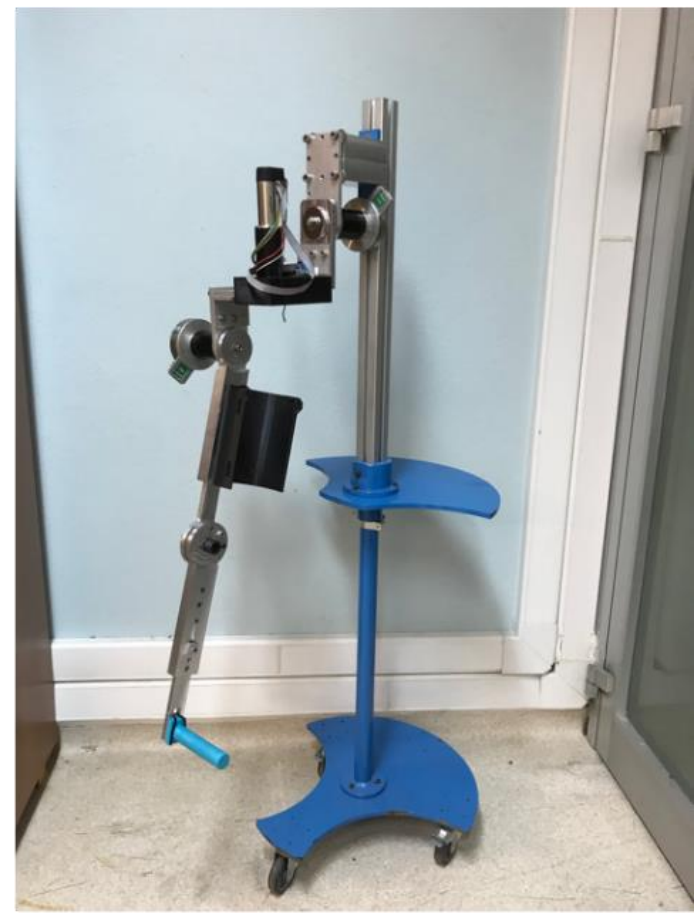

(b)

Figure 2. a) Mechanical design, b) produced prototype

\subsection{Electronic hardware}

The system block diagram can be seen in Fig.3. Raw muscle signals are taken from patients arm by Rapidan Tester branded surface electrodes. These signals are transmitted with surface electrodes to EMG circuit. In EMG circuit, these signals are amplified, filtered and rectified respectively. Raw muscle signals are come out from the EMG circuit as muscle activation levels. Force applied by the patient when performing manual exercises are measured by two force sensors that are both tension-compression micro load cell CZL635 from robot manipulator. Then, muscle activation levels and measured force data are transmitted to main controller by PCI-6025E DAQ which has 16 channels (eight differential) of analog input with 12-bit resolution, two channels of analog output with 12-bit resolution, a 100-pin connector, and 32 lines of digital I/O. The sampling time is $1 \mathrm{~ms}$. Moreover, torque and velocity data are transmitted to motor drivers (ESCON) by DAQ from main controller. According to these data, three brushless dc motors are actuated by motor drives. Maxon 
EC-Flat 60(100 Watt), EC-Max 30(40 Watt) and EC-Flat 45(30 Watt) are used as motors and ESCON 50/5 is used as motor controller. In response to the rotational movement of these motors' shafts, digital electrical signals are generated by incremental encoders (integrated with motor). Feedback is provided by the encoder to determine the position and direction information by monitoring the instantaneous positions of the motor shaft. Position information is sent to main controller by these incremental encoders by generating square signals in instant position. Then; position, force, and muscle activation level information are combined in MATLAB \& Simulink inside the main controller. Furthermore, the rehabilitation robot is used conveniently by the humanmachine interface in the main controller. Therefore, control of the manual exercises is done easily by the physiotherapists with the use of the human-machine interface. Additionally, patients are monitored in real time by physiotherapists during the manual exercises.

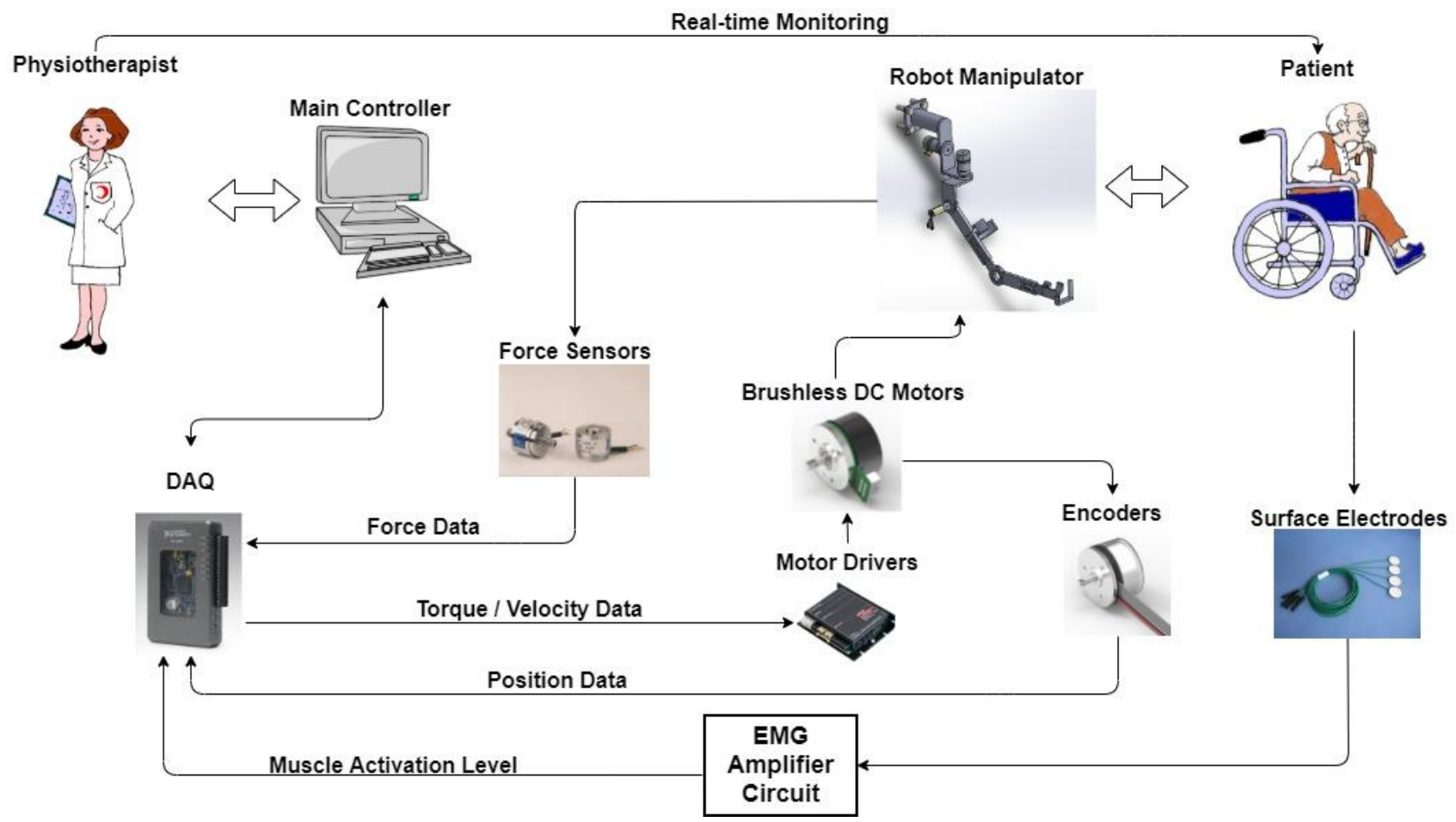

Figure 3. Block diagram of system

The motors were selected considering the load capacity and torque calculations. Mobility and angles of the human body were evaluated during the selection. In addition to this, there must be sufficient motor velocity and acceleration to execute elbow and shoulder movements. Detailed information about the motor-gearencoder specifications are summarized in Table 3.

Table 3. Motor - Gear - Encoder - Driver Specifications

\begin{tabular}{c|ccc}
\hline & Motor 1 & Motor 2 & Motor 3 \\
\cline { 1 - 2 } Motor & EC-flat 60, 100 Watt & EC-max 30, 40 Watt & EC-flat 45, 30 Watt \\
\cline { 1 - 1 } Gear & GP-52 C 52 mm, 4-30Nm & GP-32 C 32 mm, 1.0-6.0 Nm & GP-42 C 42 mm, 3-15 Nm \\
\cline { 1 - 1 } Encoder & Encoder MILE, 512 CPT & Encoder HEDL 5540, 500 CPT & Encoder MILE, 512 CPT \\
\cline { 1 - 1 } Driver & & ESCON 50/5 4-Q Servocontroller & \\
\hline
\end{tabular}

Force sensors are needed for the force-controlled exercises such as active assistive. The locations of the sensors which can be seen in Fig.4 are appropriate and calculated for all exercise types. The required range on the system is between $10 \mathrm{~kg}-15 \mathrm{~kg}$ according to exercises, so approximately range is between $98,1 \mathrm{~N}-$ $147,15 \mathrm{~N}$. Moreover, range of load cell which selected is between $0 \mathrm{~kg}-20 \mathrm{~kg}$, so its range is approximately 0 $\mathrm{N}-196,2 \mathrm{~N}$. Furthermore, according to technical requirements, length of the load cell should be less than 100 
$\mathrm{mm}$. The length of the selected load cell is $55 \mathrm{~mm}$, so Micro Load Cell CZL635 brand force sensor which is shown in Fig. 4.

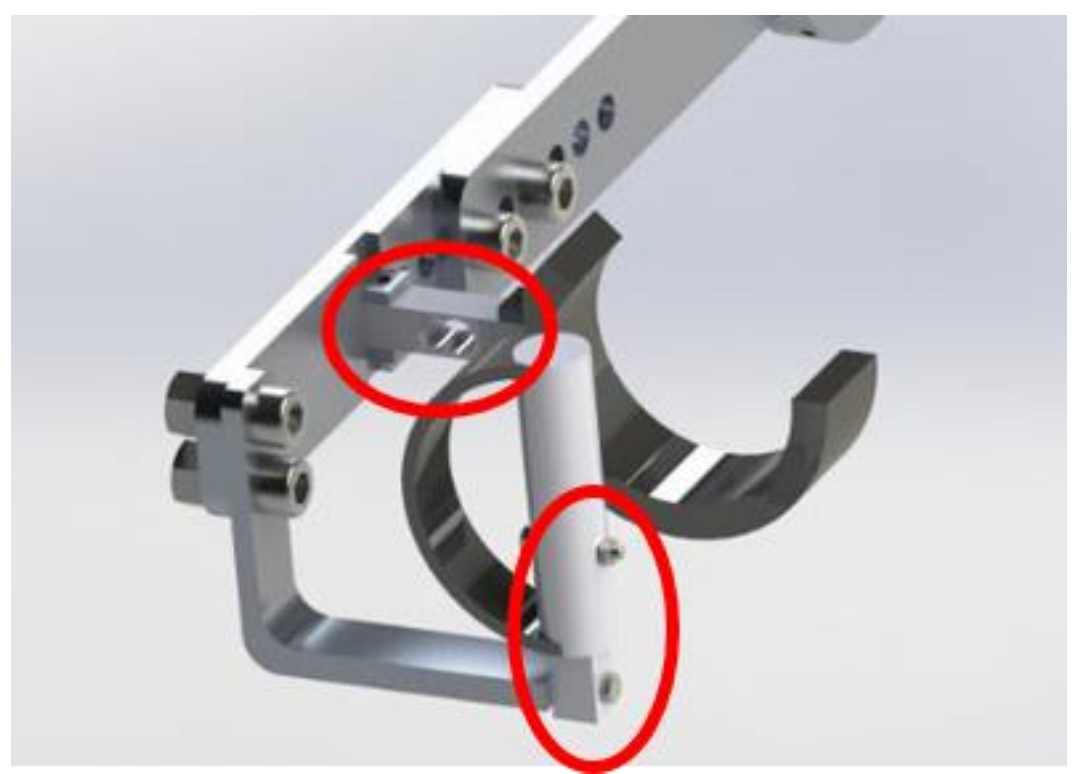

Figure 4. Locations of force sensors on the system

\subsection{EMG circuit design}

The block diagram of EMG signal processing in the system is shown in Fig.5. Full-wave rectifier, instrumentation amplifier, one high-pass filter and one low-pass filter are used for rectification, amplifying and filtering stages. Negative alternance of the muscle signal is turned into positive by full-wave rectifier. Therefore, the entire signal is fallen within the positive voltage region. Moreover, muscle signal is recovered from DC offset and low frequency noise by active high pass filter. Additionally, smooth signal is produced by active low pass filter. EMG circuit consists instrumentation amplifier and six operational amplifiers. Voltage differences between electrodes are measured by instrumentation amplifier.

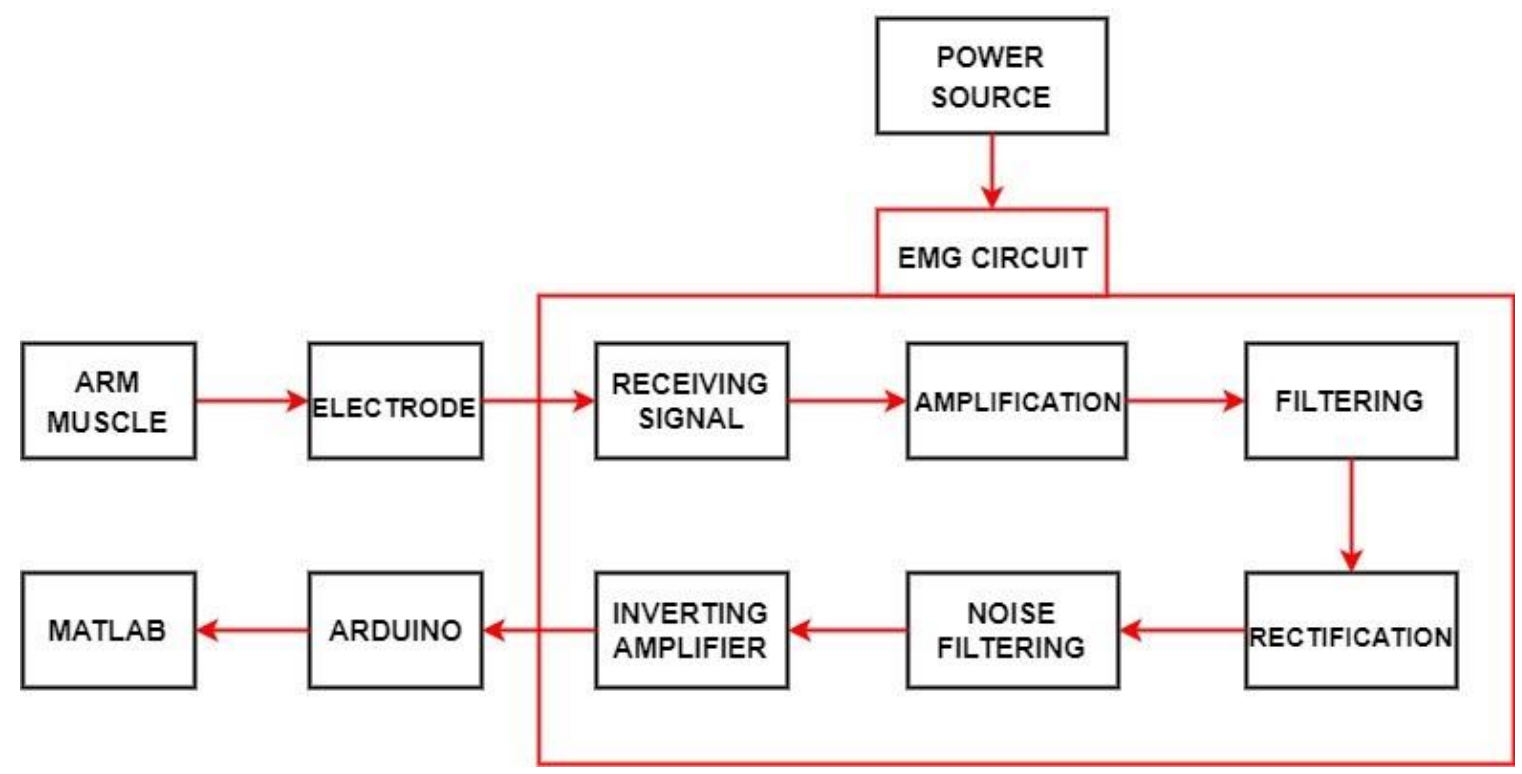

Figure 5. Block diagram of EMG circuit

EMG is used in this system for more effective control. All electronic components of EMG circuit such as OPAMPs, diodes, resistors, capacitors were specified after mathematical calculations. For EMG circuit: 2x 9V DC batteries; $2 \mathrm{x} 1 \mu F$ Tant, $1 \mathrm{x} 1 \mu F$ Ceramic Disc and $1 \mathrm{x} 0.01 \mu F$ capacitors; $1 \mathrm{x} 1 \mathrm{kohm}, 6 \mathrm{x} 10 \mathrm{kohm}, 2 \mathrm{x} 82$ kohm, 1x 100 kohm, 3x 150 kohm, 2x 1Mohm resistors;1xNA106 IC Chip, 3xTL072 IC Chip, 2x N4148 diode are used. The designed and produced EMG circuit can be seen in Fig.6.a and an experimental result 
about EMG-force relationship for resistive motion shown in Fig. 6.b. As can be seen from Fig.6.b. EMG voltage level also increases while joint force increases.

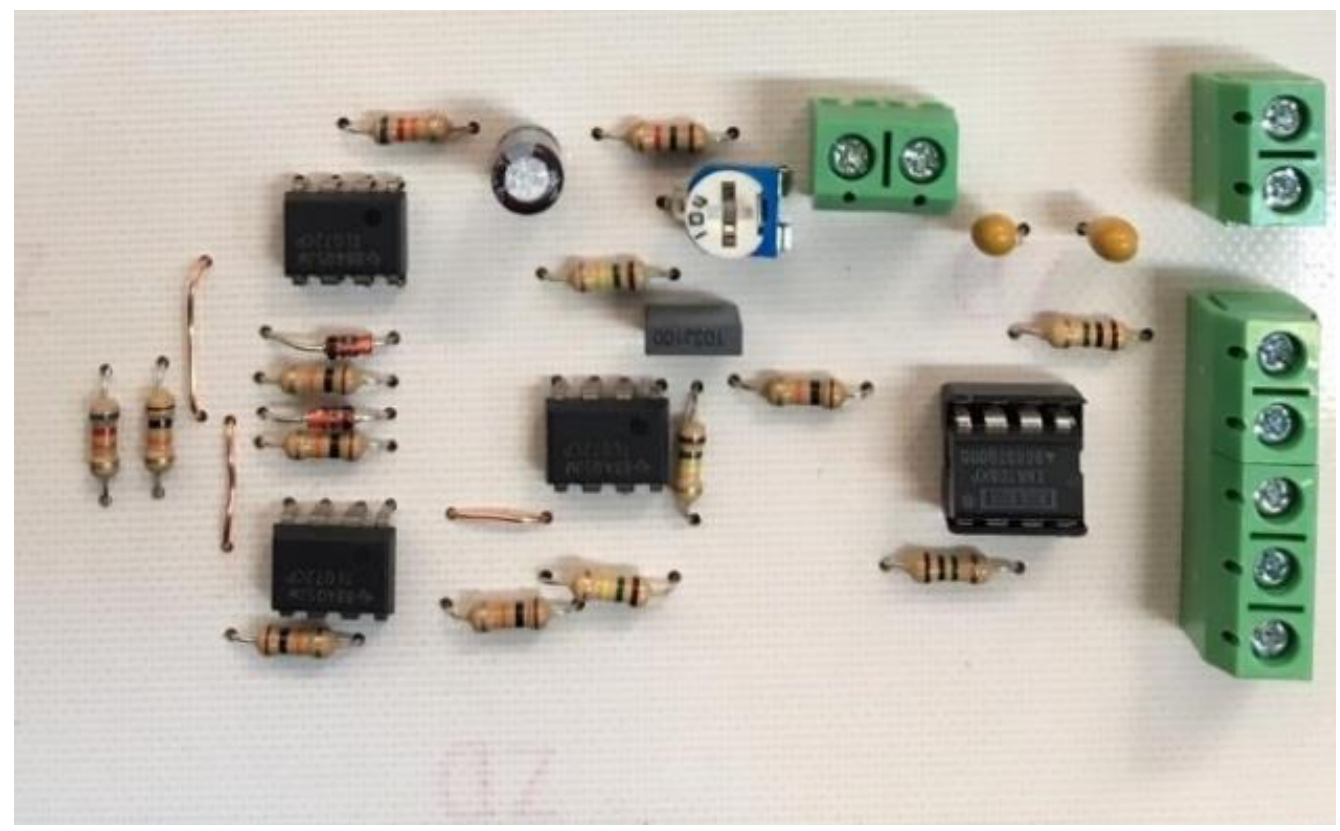

(a)

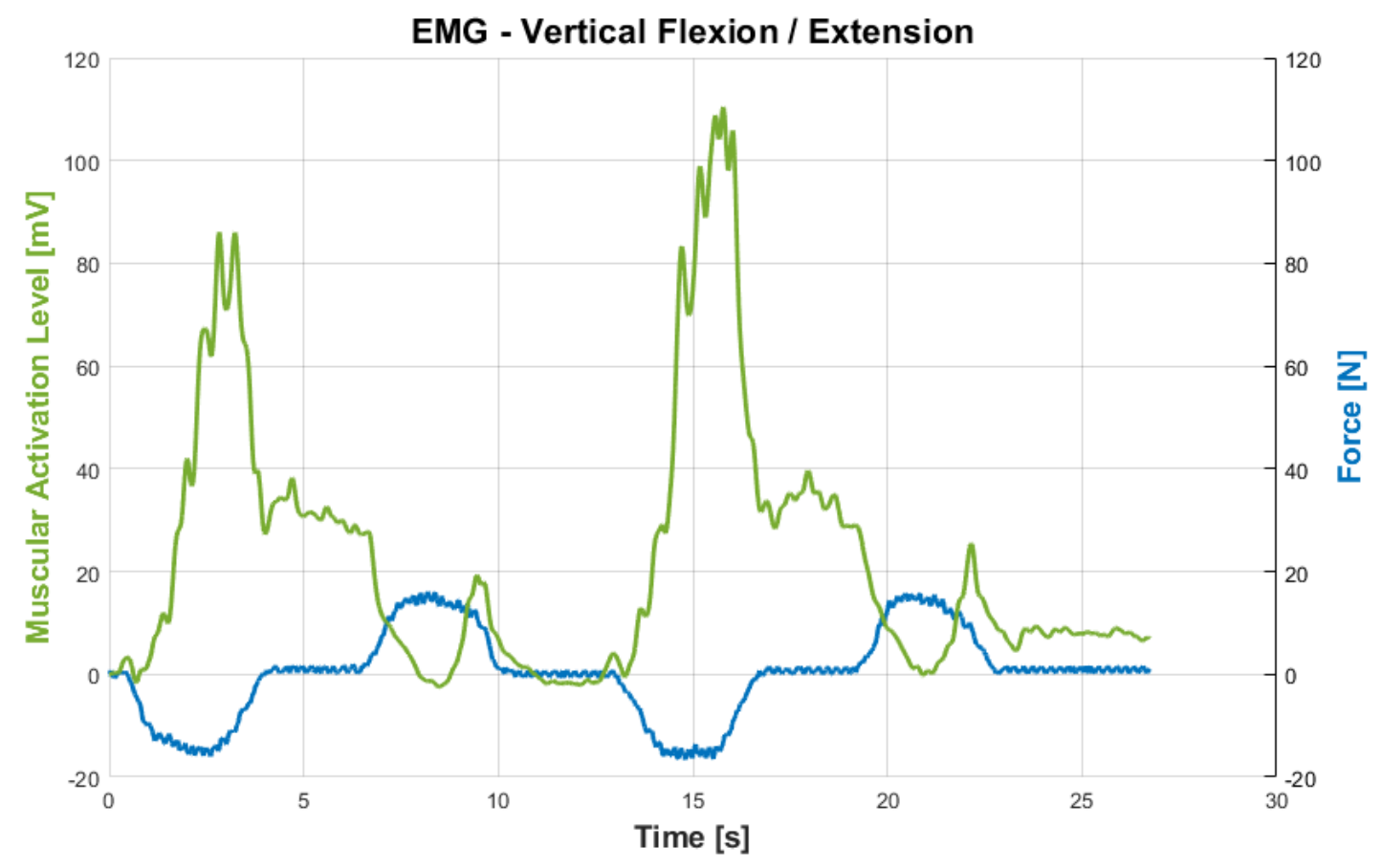

Figure 6. a) EMG circuit, b) EMG-Joint Force Relationship

\subsection{Kinematic analysis}

The link-frame attachment in Fig. 7 is used to build-up the kinematic model of the rehabilitation robot. In this model joints 0,1 and 2 together form the glenohumeral joint, where joint 0 corresponds to abductionadduction, joint 1 horizontal flexion-extension and joint 2 to vertical flexion-extension. Joint 3 , which fits to flexion-extension of the elbow joint is located outside the shoulder joint. 


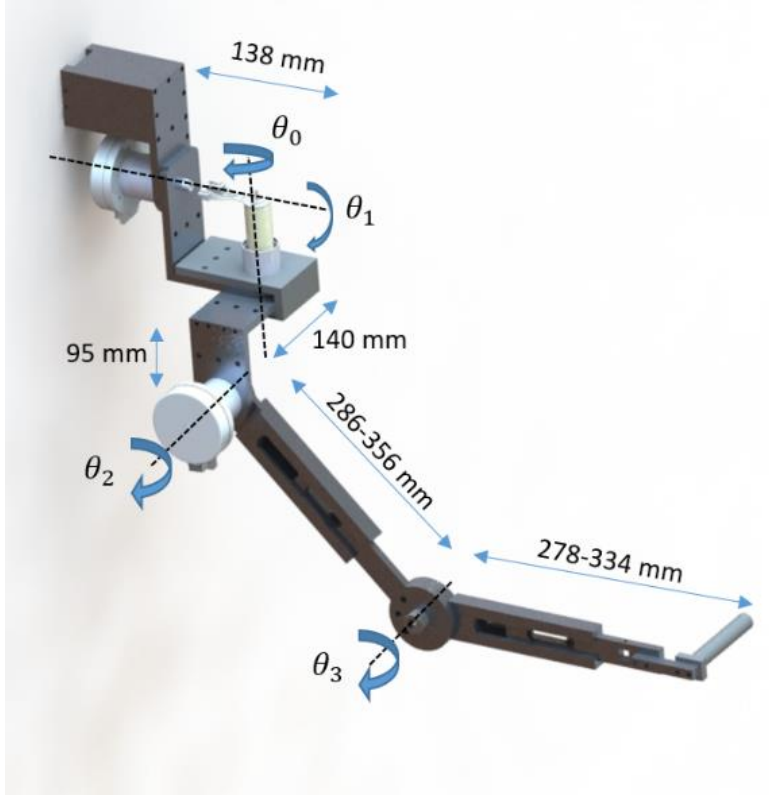

Figure 7. Link - frame attachments

The DH (Denavit-Hartenberg) parameters are summarized in Table 4.

Table 4. Denavit - Hartenberg parameter table

\begin{tabular}{c|cccc}
$i$ & $a$ & $\alpha$ & $d$ & $\theta$ \\
\hline 1 & $a_{1}$ & $\pi / 2$ & 0 & $\theta_{0}$ \\
\cline { 1 - 1 } 2 & $a_{2}$ & $\pi / 2$ & $d_{1}$ & $\theta_{1}$ \\
\cline { 1 - 1 } & $a_{3}$ & 0 & 0 & $\theta_{2}$ \\
\cline { 1 - 1 } & $a_{4}$ & 0 & 0 & $\theta_{3}$ \\
\hline
\end{tabular}

\subsection{Dynamic analysis}

Euler - Lagrange method is used to determine the dynamics of the rehabilitation robot. This method handles both the joint velocities and position of the system together.

With this data potential energy and kinetic energy of the system can be found easily. For certain restriction class systems, it generalizes the Newtonian mechanics. During dynamic analysis, methods in the studies of Akdoğan et al. [29] and Riani et al. [19] were taken into consideration.

In this study, all three motors allow the system to move in 2 axes. Motor 1, motor 2 and motor 3 provide the system to move in $\mathrm{Y}-\mathrm{Z}$ axes, $\mathrm{X}-\mathrm{Z}$ axes, $\mathrm{X}-\mathrm{Y}$ axes. Therefore, three motors are accepted as pendulum systems. Equation (1) represents the dynamic model of a pendulum which can be seen in Fig.8.

$$
\begin{aligned}
& M(q) \ddot{q}+h_{N}(q, \dot{q})=\tau \\
& h_{N}(q, \dot{q})=C(q, \dot{q})+G(q)
\end{aligned}
$$

where $\tau$ is joint torque, $q$ is joint angle, $M(q)$ is inertia matrix, $C(q, \dot{q})$ is Coriolis and centrifugal matrix and $G(q)$ is gravitational matrix.

$$
\begin{aligned}
& M(t)=M_{N}(t)+M_{\Delta}(t) \\
& h(q, \dot{q})=h_{N}(q, \dot{q})+h_{\Delta}(q, \dot{q})
\end{aligned}
$$




$$
\tau(t)=\tau_{N}(t)+\tau_{\Delta}(t)
$$

where $M_{N}(t), h_{N}(q, \dot{q})$ and $\tau_{N}(t)$ are represented as nominal parts of $M(t), h(q, \dot{q})$ and $\tau(t)$. Because of the slow movements are performed in the system, $C(q, \dot{q})$ is neglected. Therefore,

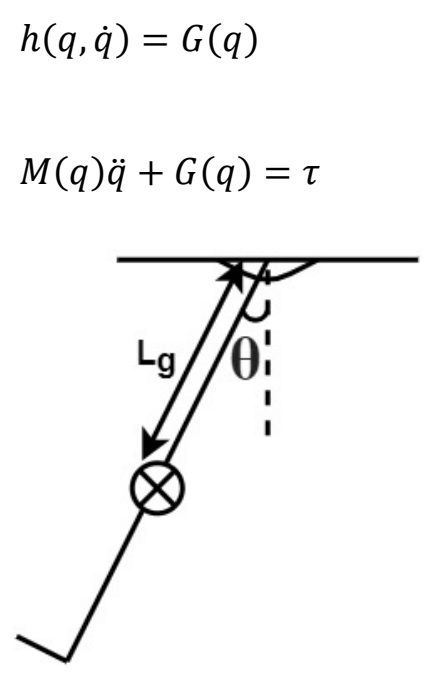

Figure 8. Pendulum model

According to three motors, $L_{g}$ is the distance of the link mass center to the rotation point. $L_{g_{1}}(r e d)$, $L_{g_{2}}$ (green) and $L_{g_{3}}$ (blue) are the distance of the link mass center to the motor 1, motor 2 and motor 3 as can be seen in Fig. 9. Equation (8) represents the dynamic model of a pendulum.

$$
M \ddot{\theta}+m g l \sin \theta+F(\theta, \dot{\theta})=\tau
$$

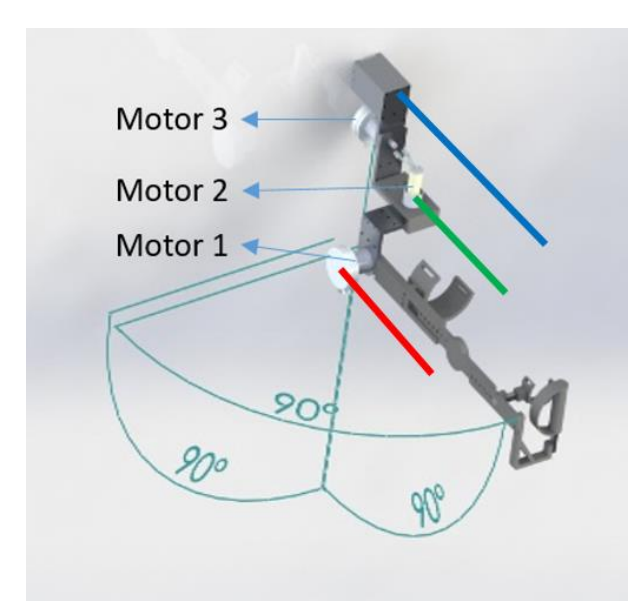

Figure 9. Location of motors and joint angles

where $\tau$ is torque, $M$ is inertia, $m$ is mass, $g$ is gravitational acceleration constant, $l$ is length of pendulum and $\theta$ is angle of pendulum. Coriolis and fraction forces $(F(\theta, \dot{\theta}))$ are neglected because of slow-motion of the system. The dynamic equation of the system for a joint is given in (9) and location of motors is shown in Fig. 8.

$$
\theta(s)\left[M(s) s^{2}+m g l \frac{1}{\left(s^{2}+1\right)}\right]=\tau(s)
$$

The transfer function for joint angle 1 is given in (10). 


$$
\frac{\theta_{1}(s)}{\tau(s)}=\frac{s^{2}+1}{0,160 s^{2}\left(s^{2}+1\right)+7,62}
$$

where $\theta_{1}$ is shaft angle of motor 1 . The transfer function for joint angle 2 is given in (11).

$$
\frac{\theta_{2}(s)}{\tau(s)}=\frac{1}{0,961 s^{2}}
$$

where $\theta_{2}$ is shaft angle of motor 2. The transfer function for joint angle 3 is given in (12).

$$
\frac{\theta_{3}(s)}{\tau(s)}=\frac{s^{2}+1}{0,343 s^{2}\left(s^{2}+1\right)+22,07}
$$

where $\theta_{3}$ is shaft angle of motor 3 .

\subsection{Control methods and exercise types}

\section{PID Control:}

Exercise types and related control methods are shown in Tab. 5. PID control method ensures the ability to use the three control terms of the proportional, integral and derivative effect on the controller output to apply accurate control. Systematic parameter exchange method to determine PID parameters was used on the rehabilitation robot. The PID Control law is given in (13).

$$
G_{P I D}(s)=K_{p}+\frac{K_{I}}{s}+K_{d} s
$$

where $K_{p}, K_{d}, K_{i}$ represents proportion constant, derivative constant and integral constant, respectively.

Table 5. Exercise types \& control methods

\begin{tabular}{cc}
\hline Exercise Types & Control Methods \\
\hline Passive & PID Control \\
Active-assistive & Force and Position Based Impedance Control \\
Isometric & Force Based Impedance Control \\
Isotonic & Force Based Impedance Control \\
\hline
\end{tabular}

\section{Impedance Control:}

In rehabilitation systems impedance control method is used extensively because of effectiveness in humanrobot interaction systems [30]. Force and position can be used together or separately in different impedance control methods by tuning the mechanical impedance of the end effector of the robot. The regulation of force or position is maintained in a complex way. The relationship between force and position is regulated in one side meanwhile, velocity and acceleration are regulated on the other side. It needs position as an input and the output will be a resulting force. Mechanical impedance is the response of mechanism elasticity against external force applied. Mechanical impedance is adjustable and can be changed during the exercises while the robot tracks the desired force or position continuously. Therefore, impedance control tends to be position based or force based. Force based impedance control method is suitable for isometric and isotonic exercise since desired force is required. Because of the both position and force control are necessary for activeassistive exercise, both position and force-based impedance controller are used.

The robot dynamic equation is expressed by the following nonlinear equation:

$$
\tau=M(q) \ddot{q}+C(q, \dot{q})+G(q)+F(\dot{q})-J^{T}(q) F_{e}
$$


where $M(q)$ is inertia, $C(q)$ is the Coriolis and centrifugal, $G(q)$ is gravitational and $J(q)$ is the Jacobian matrices. $q$ is the angular position vector. $F_{e}$ is the external force applied by the patient. $F(\dot{q})$ represents the friction forces.

For position-based impedance control;

The desired dynamic behaviour of the robot manipulator after applying position-based impedance control can be given as:

$$
M_{d}\left(\ddot{x}-\ddot{x_{d}}\right)+B_{d}\left(\dot{x}-\dot{x_{d}}\right)+K_{d}\left(x-x_{d}\right)=-F_{e}
$$

where $M_{d}, B_{d}$ and $K_{d}$ are symmetrical matrices that represents desired inertia, damping and stiffness matrices. The vector $x$ denotes the end-effector position and orientation, and $x_{d}$ represents the desired end-effector position and orientation.

$$
\ddot{x}=\ddot{x_{d}}+M_{d}^{-1}\left[-B_{d}\left(\dot{x}-\dot{x_{d}}\right)-K_{d}\left(x-x_{d}\right)-F_{e}\right]
$$

The velocity of the end-effector is;

$$
\dot{x}=J(q) \dot{q}
$$

And the acceleration is;

$$
\ddot{x}=J(q) \ddot{q}+J(\dot{q}) \dot{q}
$$

Consider the robot dynamics in (14) and choose the control input as;

$$
\tau=M(q) u+C(q, \dot{q})+G(q)+F(\dot{q})-J^{T}(q) F_{e}
$$

Then the dynamic equation becomes $\ddot{q}=u$ (11) becomes;

$$
\ddot{x}=J(q)(\ddot{x}-\dot{J}(q) \dot{q})=u
$$

The resulting control equation after combining these equations;

$$
\begin{gathered}
\tau=M(q) J(q)\left(\ddot{x_{d}}+M_{d}^{-1}\left[-B_{d}\left(\dot{x}-\dot{x_{d}}\right)-K_{d}\left(x-x_{d}\right)-F_{e}\right]-J(q) \dot{q}\right) \\
+C(q, \dot{q})+G(q)+F(q)-J(q)^{T} F_{e}
\end{gathered}
$$

\section{For force-based impedance control;}

For the force-based impedance control case, the desired dynamics behaviour of the system can be given as:

$$
M_{d} \ddot{x}+B_{d} \dot{x}-F_{d}=-F_{e}
$$

which is equal to:

$$
\ddot{x}=M_{d}^{-1}\left(-B_{d}\left(\dot{x}+F_{d}-F_{e}\right)\right.
$$

where

$$
\begin{gathered}
\tau=M(q) J(q)\left(M_{d}^{-1}\left[F_{d}-F_{e}-B_{d} \dot{x}\right]-j(q) \dot{q}\right)+C(q, \dot{q})+G(q)+F(q)- \\
J(q)^{T} F_{e}
\end{gathered}
$$




\section{Results and discussions}

\subsection{Strength analysis}

Approximate force and torque values were applied to the robot arm according to the maximum mass and dimensions expected from the patients who would use the system. There are two methods to obtain yield criteria (çoğulu olmaz) for ductile materials. These are Tresca and Von mises criterions. Von - Mises criterion was used in this study because Tresca criterion steers to take unnecessary measures to prevent a possible problem. In this study, weight is an important parameter.

Von Mises is based on the determination of the distortion energy in a given material. A material is safe as long as the maximum value of the distortion energy per unit volume stands smaller than the distortion energy per unit volume.

Analysis were performed with respect to the load capacity of the system. The static calculations are performed to all system from the end effector where the system meets with patient's hand. According to the strength analysis, the connection point where the robot connects to the stand needs primary consideration because of the extra bending. As a result of the calculations based on the yield strength for aluminium material, the thicknesses on the connection part are increased to make the system safer. The system is all in blue and strong enough as shown in Fig.10.

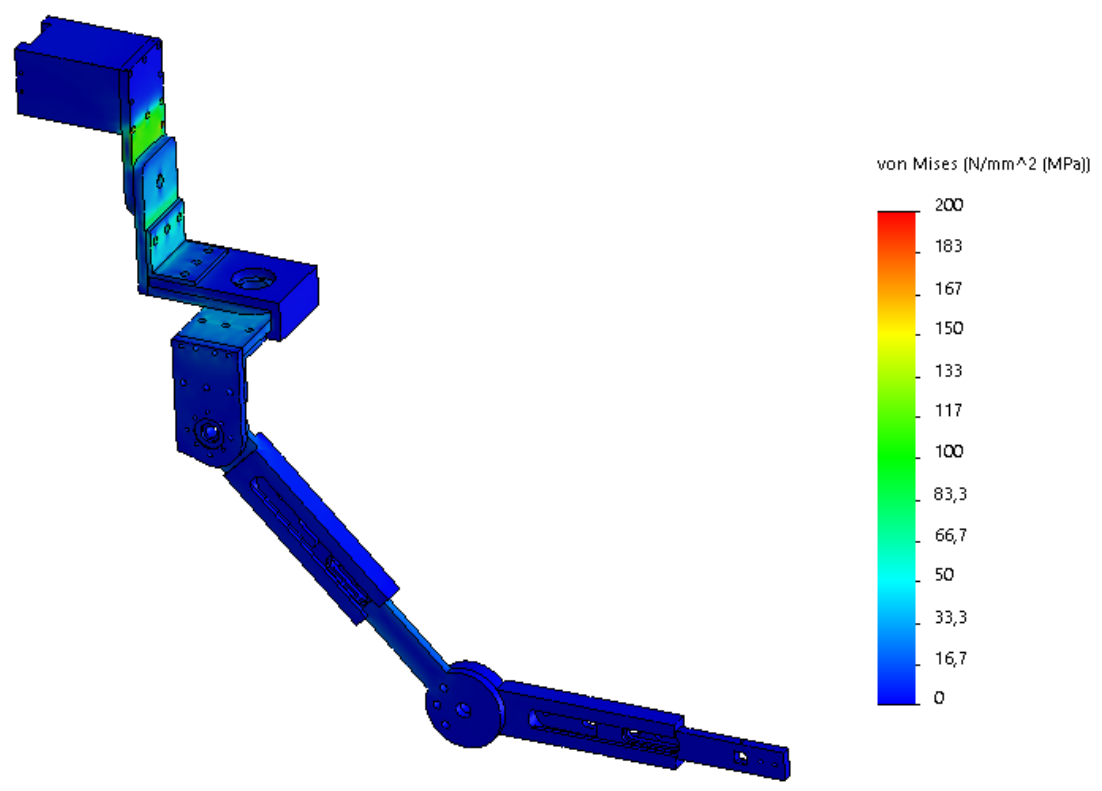

Figure 10. Strength analysis

\subsection{Simulation results with Simscape model}

The passive exercise was simulated by using PID control method for each motor. The system's mechanical 3D model was transferred from Solidworks to Simulink with the help of Simscape tool. In the Simulink environment, this model was controlled via PID control method. Two types of inputs are given to these systems. One of them was a step function and the other one was a sine function.

Step response of joint 1 is given in Fig.11.a for the step function. The robot manipulator fitted on the step wave in five seconds which was acceptable and the slope was appropriate. There is an acceptable steady state error. In Fig.11.b the response of joint 1 to the sine input can be seen. According to this figure, the robot manipulator follows the reference sinusoidal trajectory with high accuracy. 


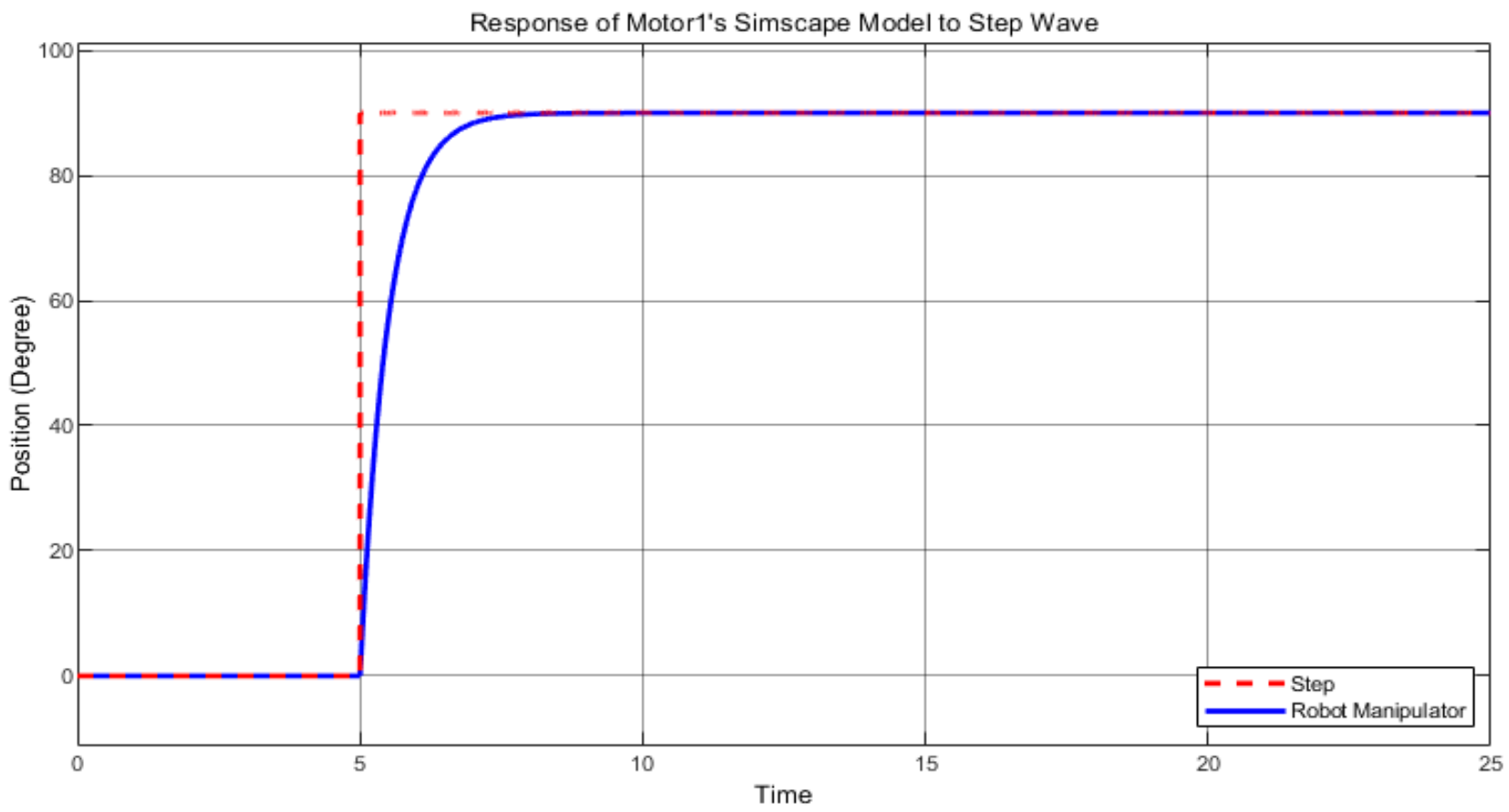

(a)

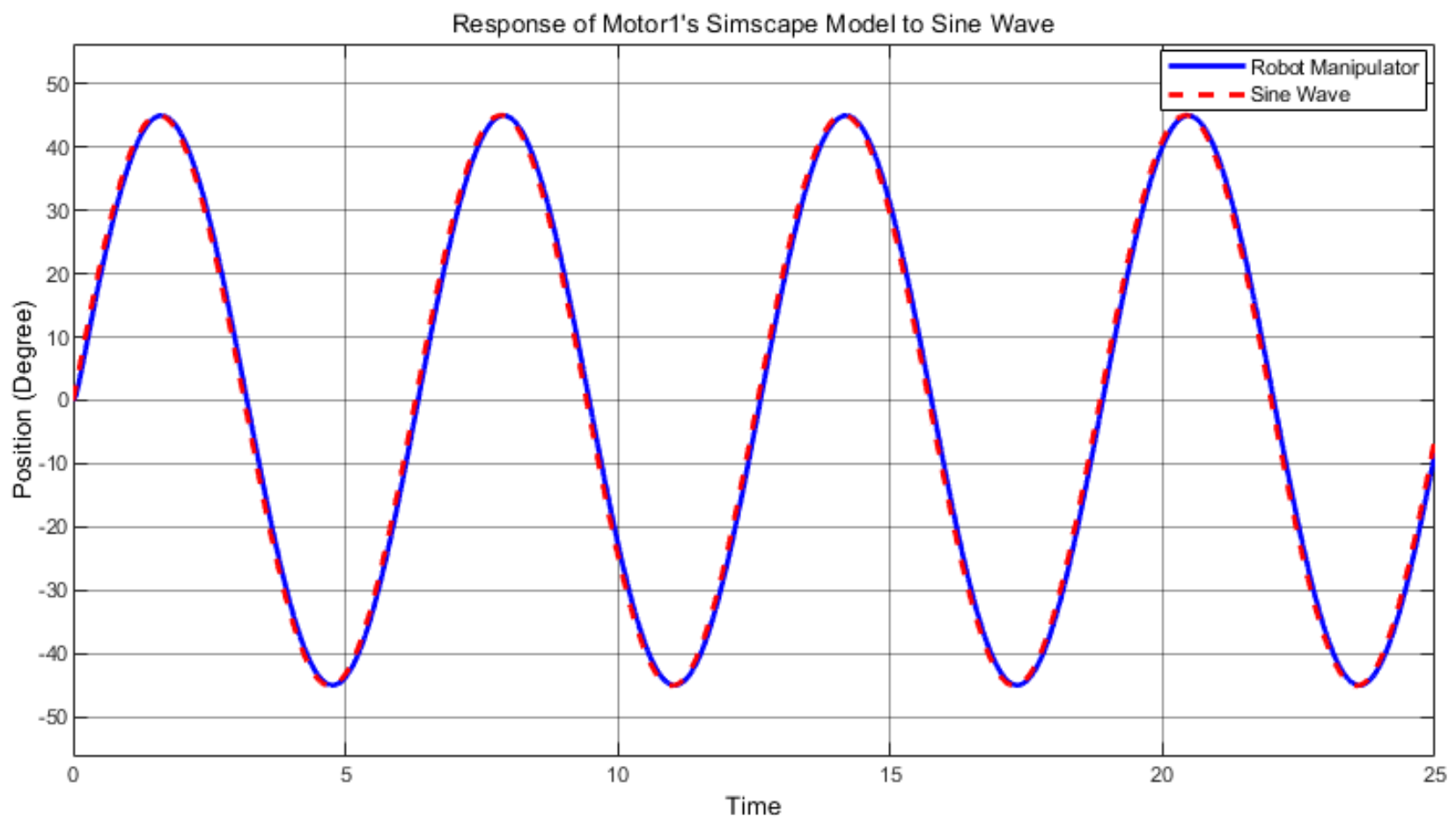

(b)

Figure 11. Joint 1 response a) step input, b) sinus input

The step response of joint 1 is shown in Fig.12.a. The robot manipulator fitted on the step wave in five seconds which is acceptable and the rising time is appropriate for application. The response of joint 1 for the sinusoidal trajectory can be seen in Fig.12.b.

According to this result, the robot manipulator follows the sinusoidal reference trajectory with high accuracy. 


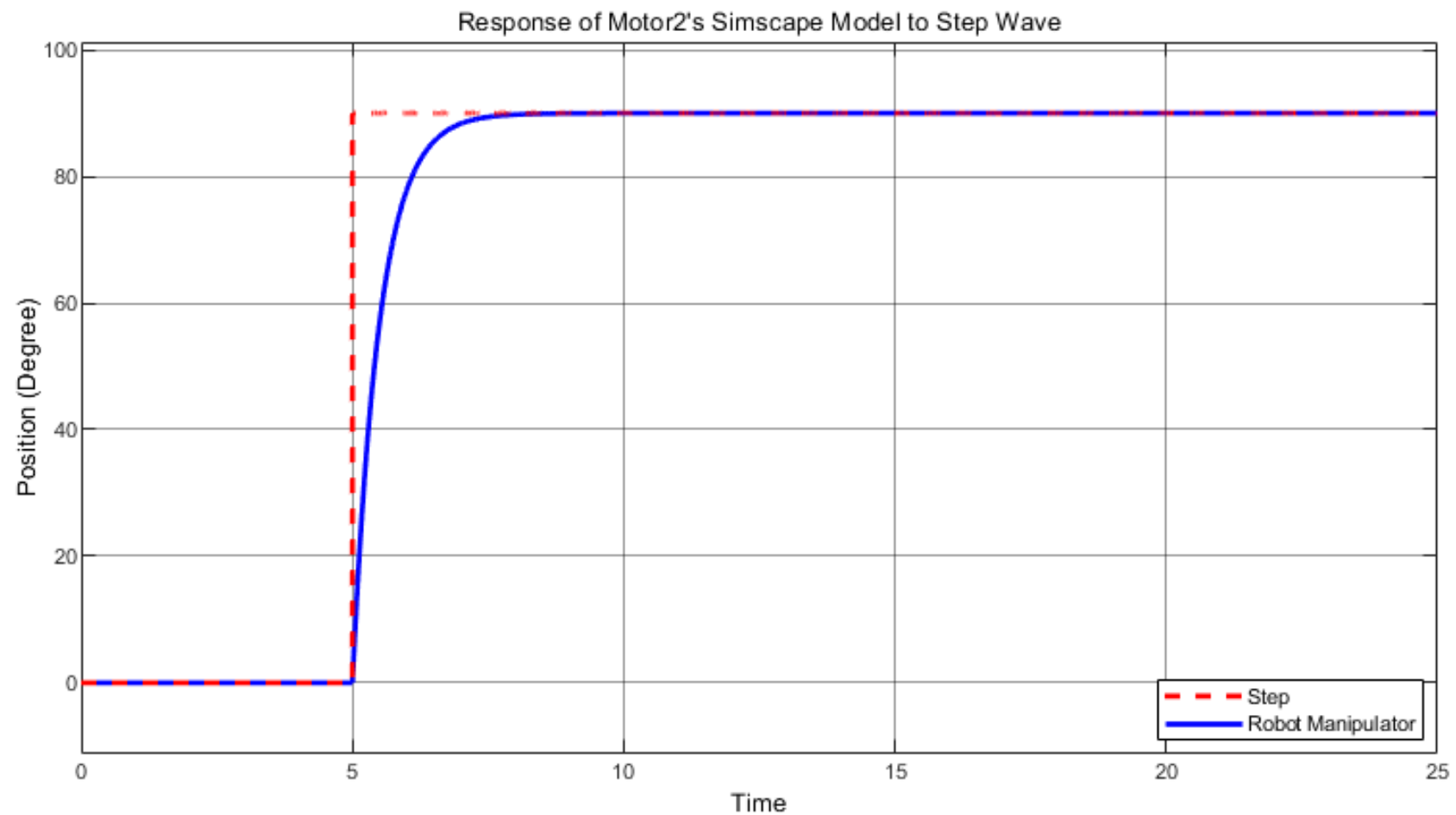

(a)

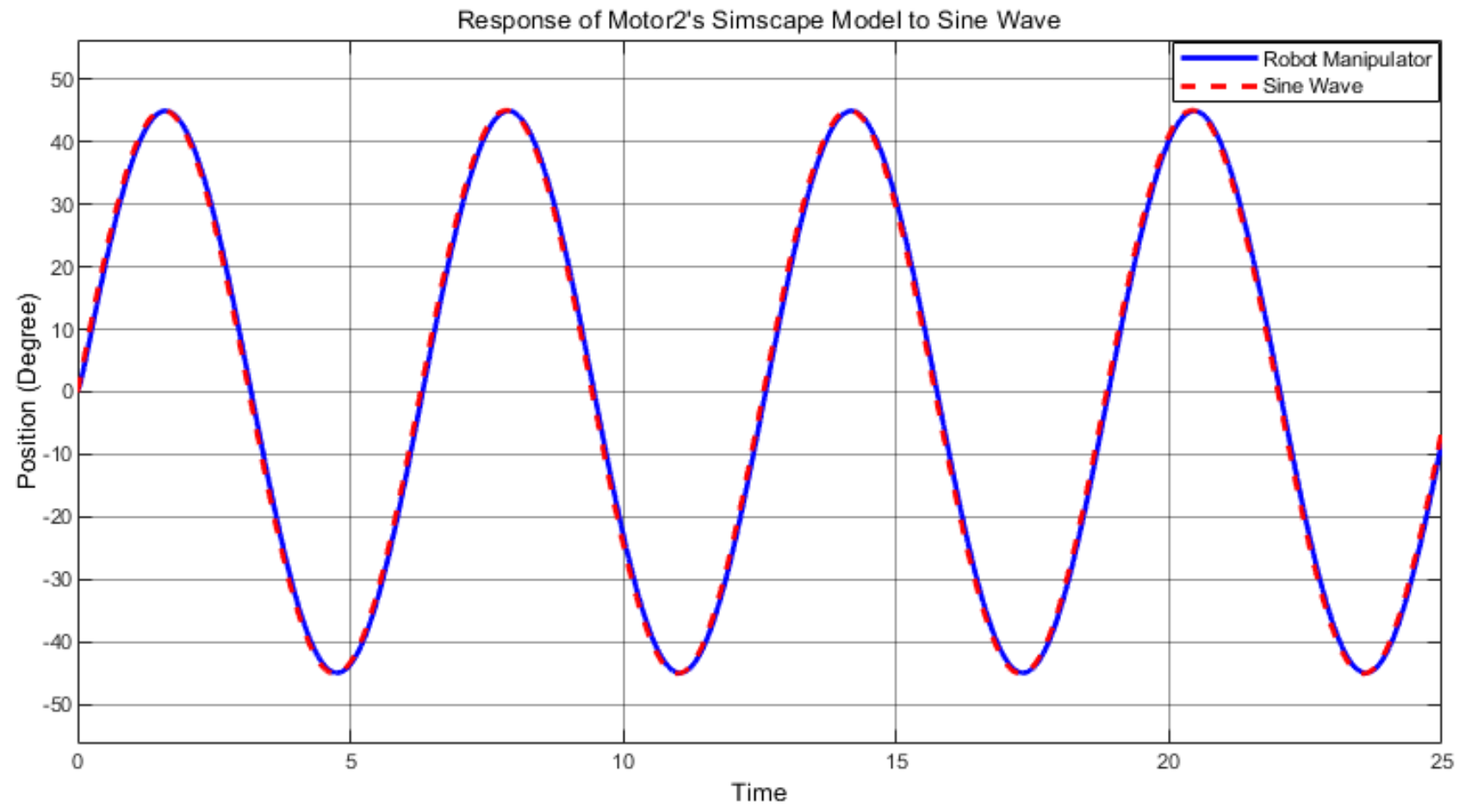

(b)

Figure 12. Joint 2 response a) step input, b) sinus input

In Fig.13.a the response of joint 1 to the step function. The robot manipulator fitted on the step wave in five seconds which was acceptable and the slope was appropriate. In Fig.13.b the response of joint 3 to the sine function can be seen. As can be seen from Fig.13.b the robot manipulator tracks the sine wave as expected. 


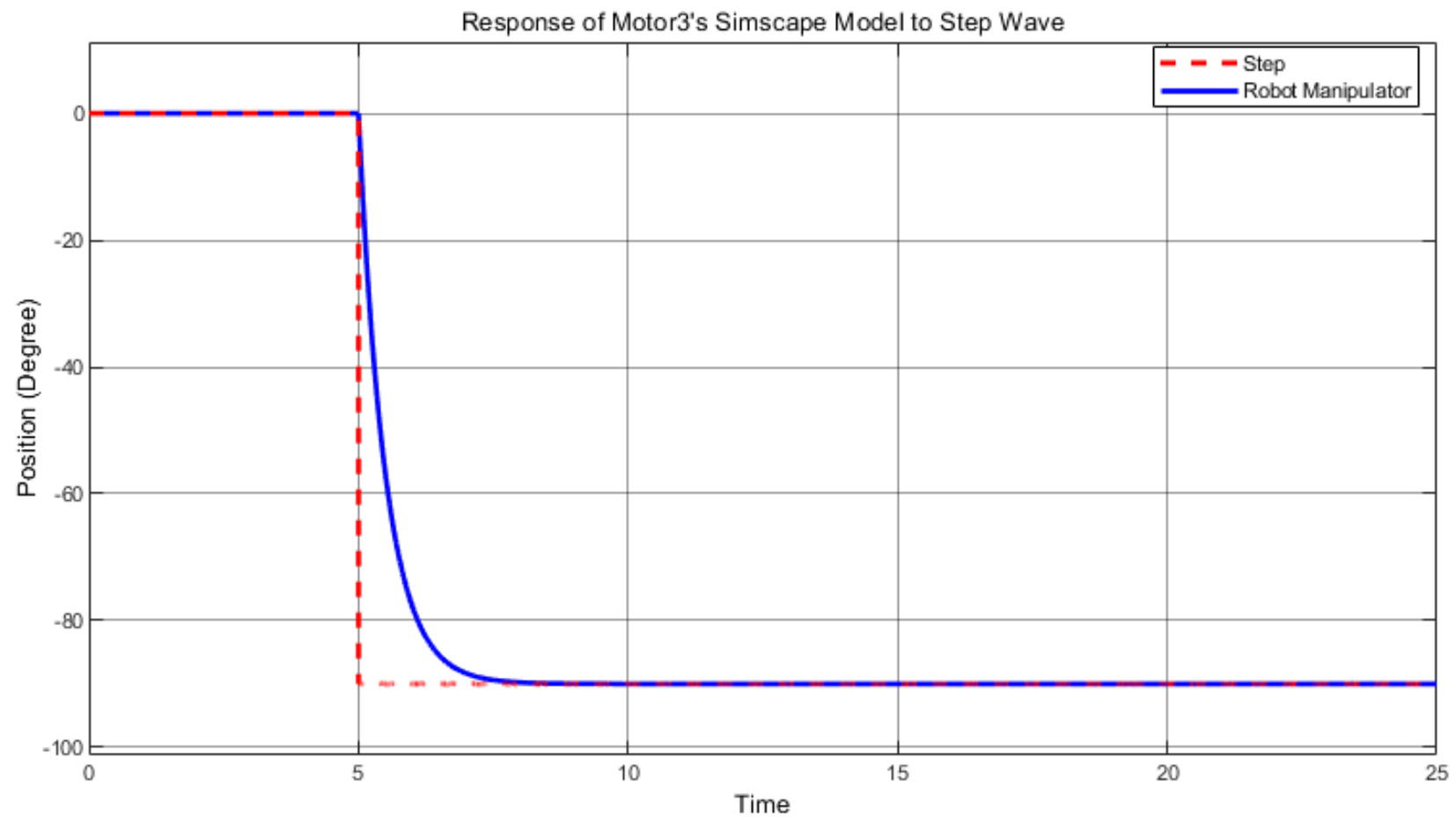

(a)

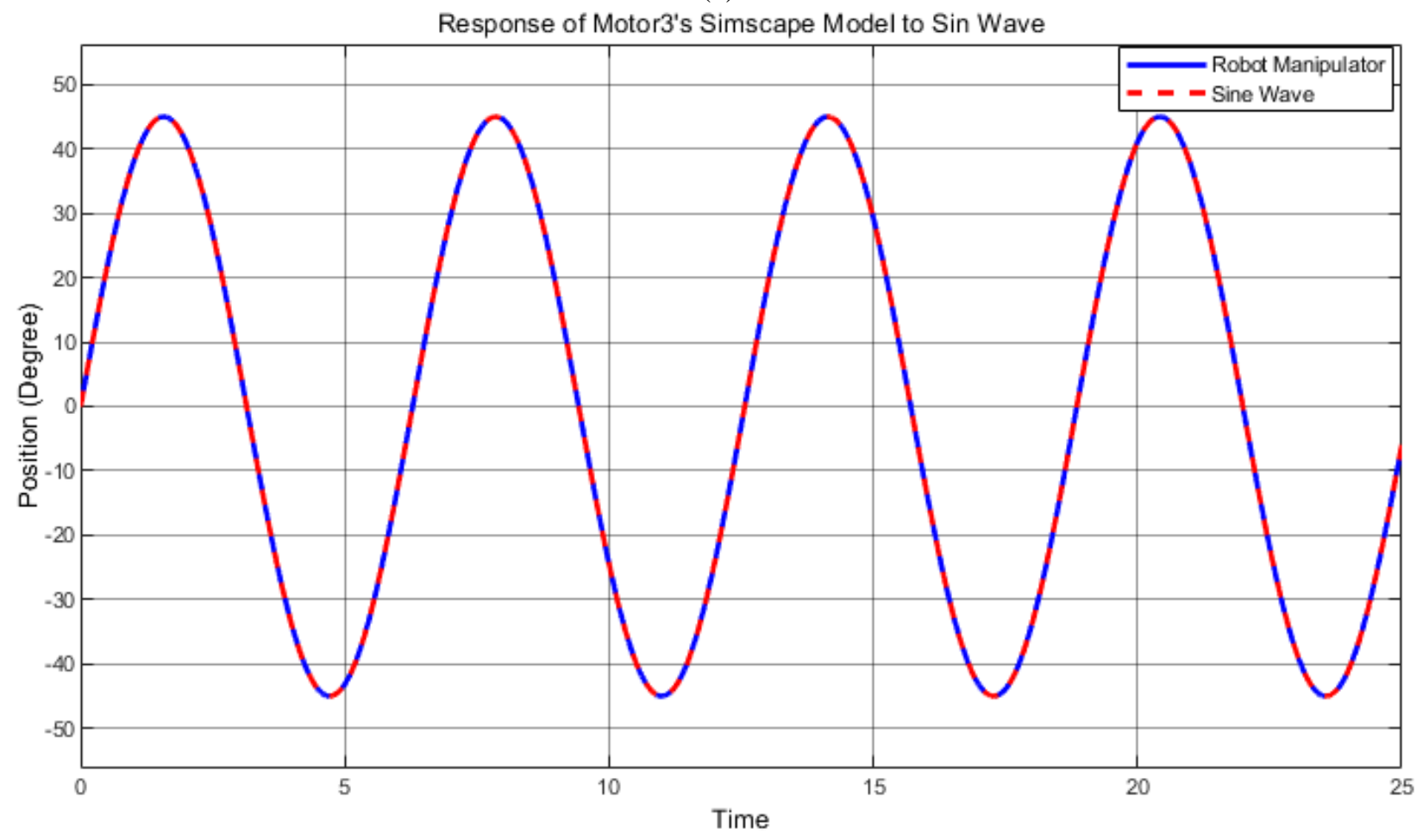

(b)

Figure 13. Joint 3 response a) step input, b) sinus input

\subsection{Simulations with dynamic models of the system}

The transfer functions of the dynamic models for all joints were placed in three separate control systems as a subsystem in the control diagrams in Simulink. The PID values were tuned by the Heuristic approach. Step and sinewave trajectory command were sent to the systems for testing and simulation. 
As shown in Fig.14 the dynamic model for motor 1 is tracking the sine wave and step wave properly without delays and vibration in simulation.

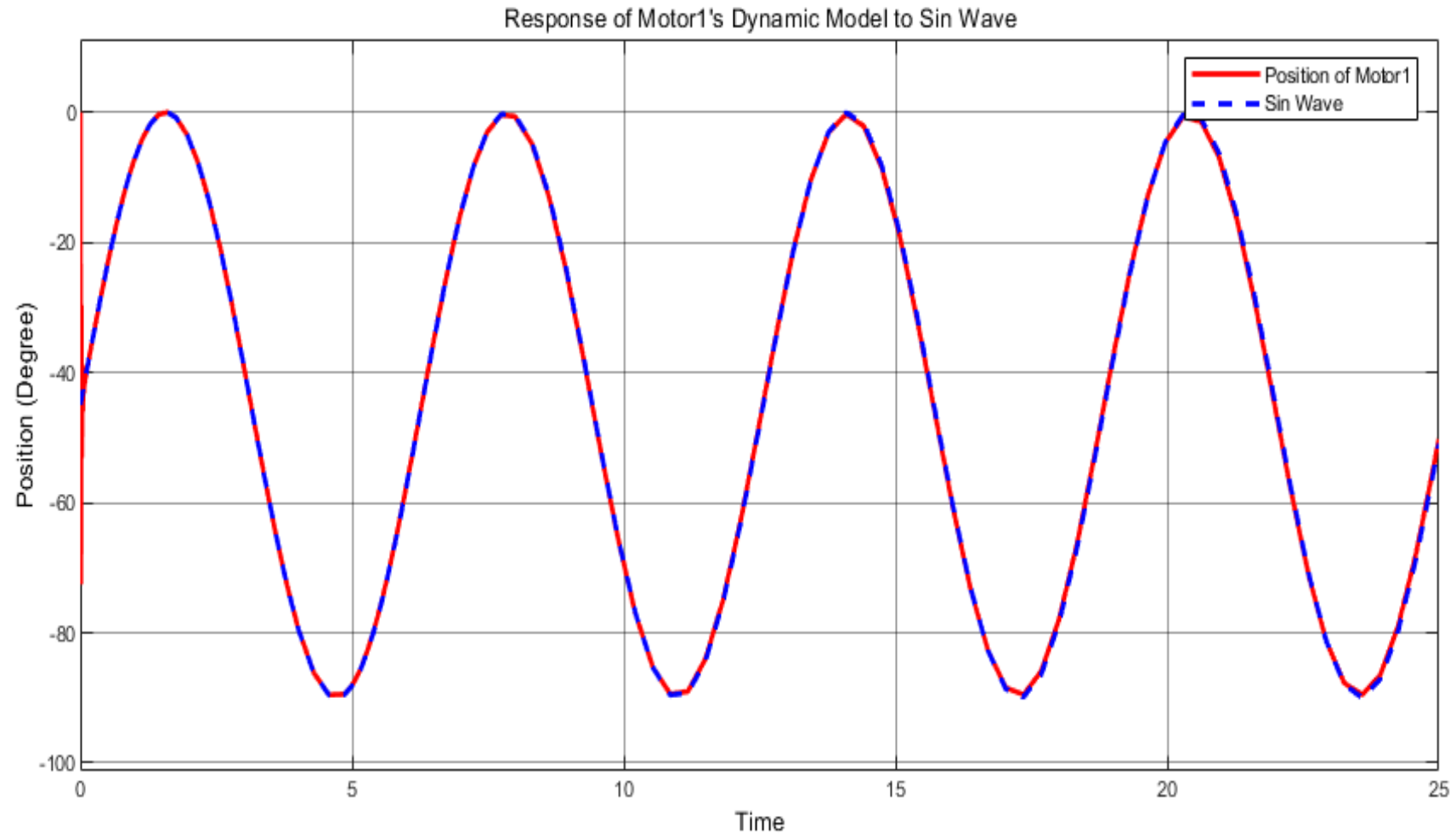

(a)

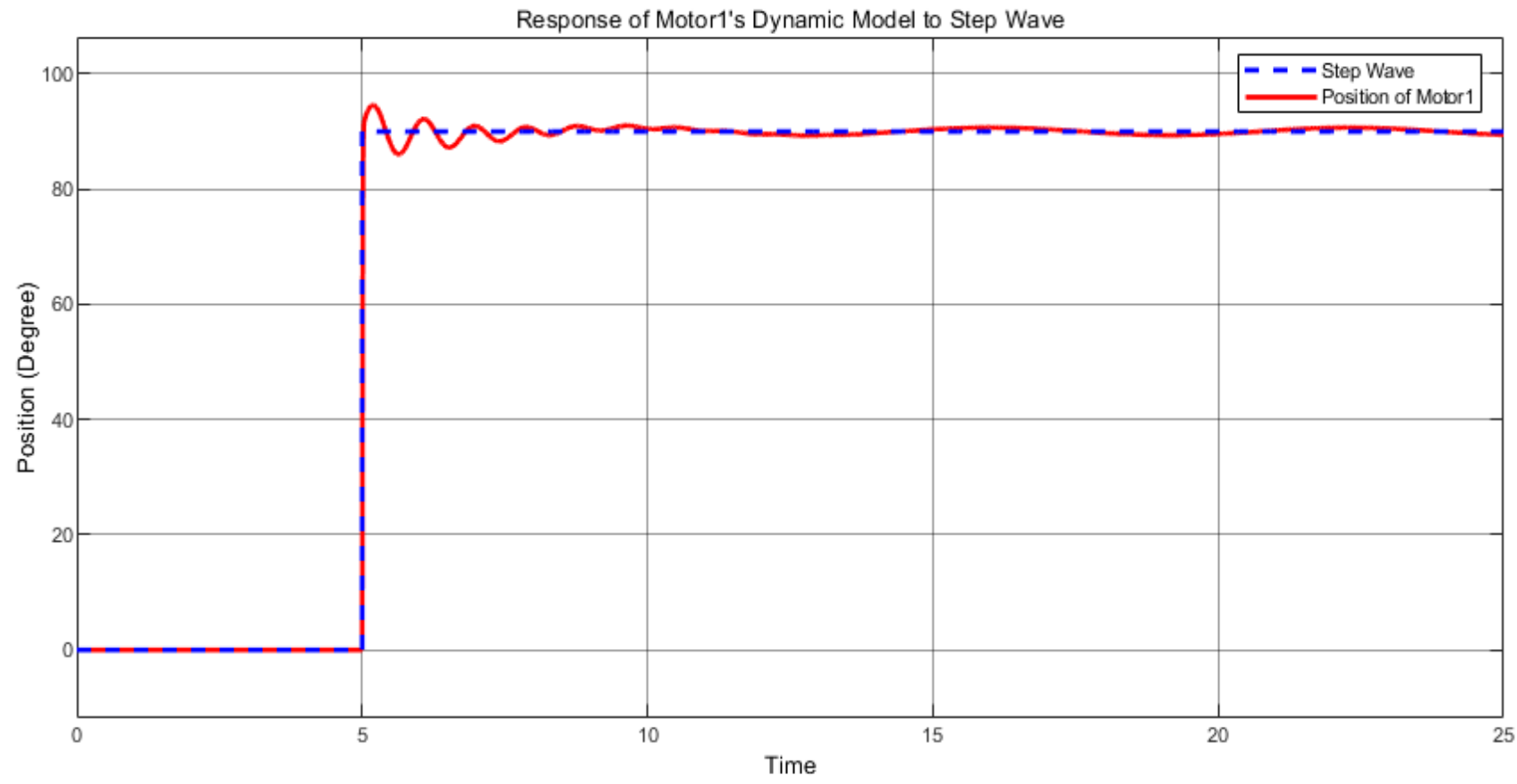

Figure 14. Joint 1 response a) sinus input, b) step input

As shown in Fig.15 the dynamic model for joint 2 is tracking the sine wave and step wave properly without delays and vibration in simulation. 


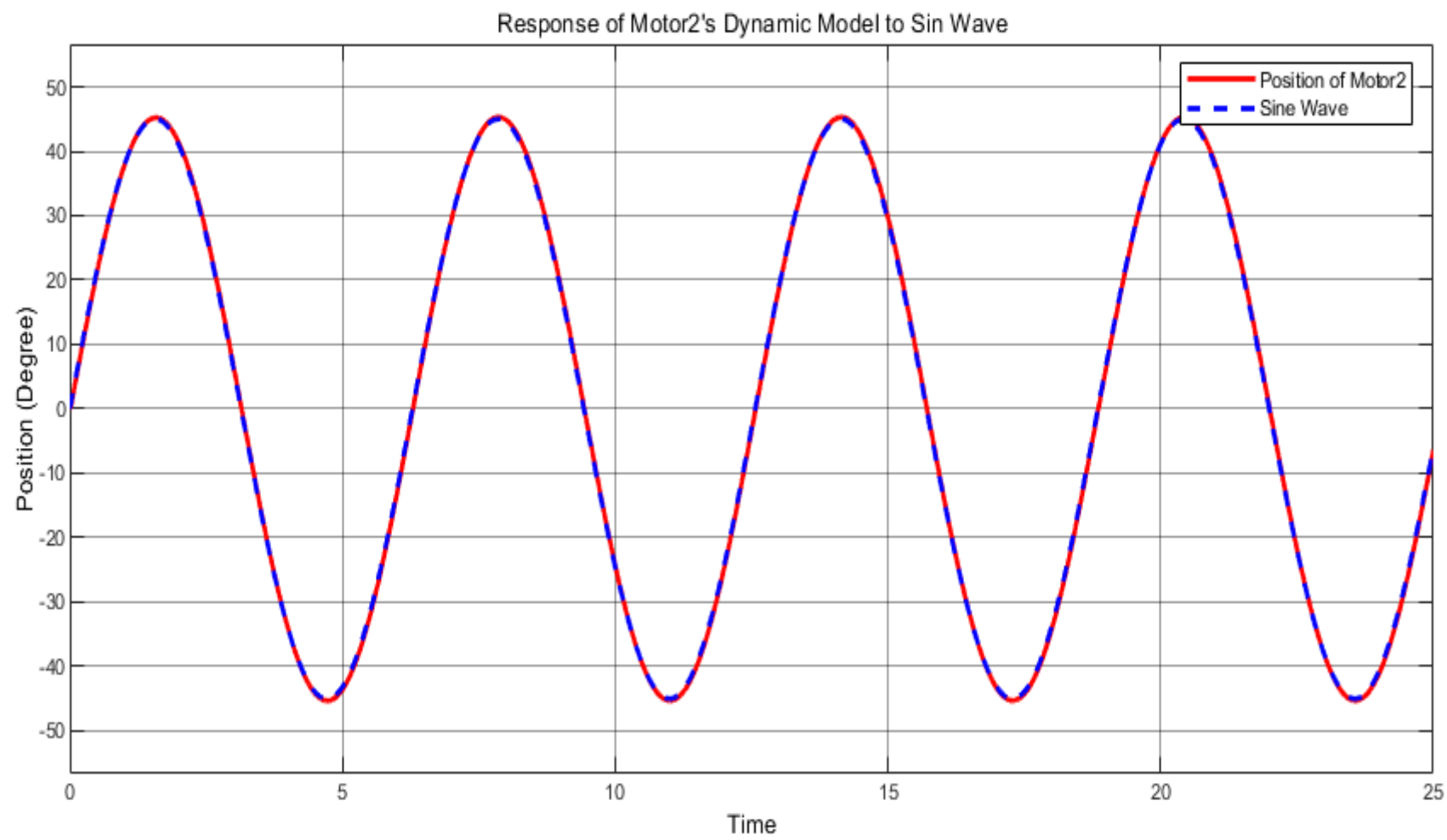

(a)

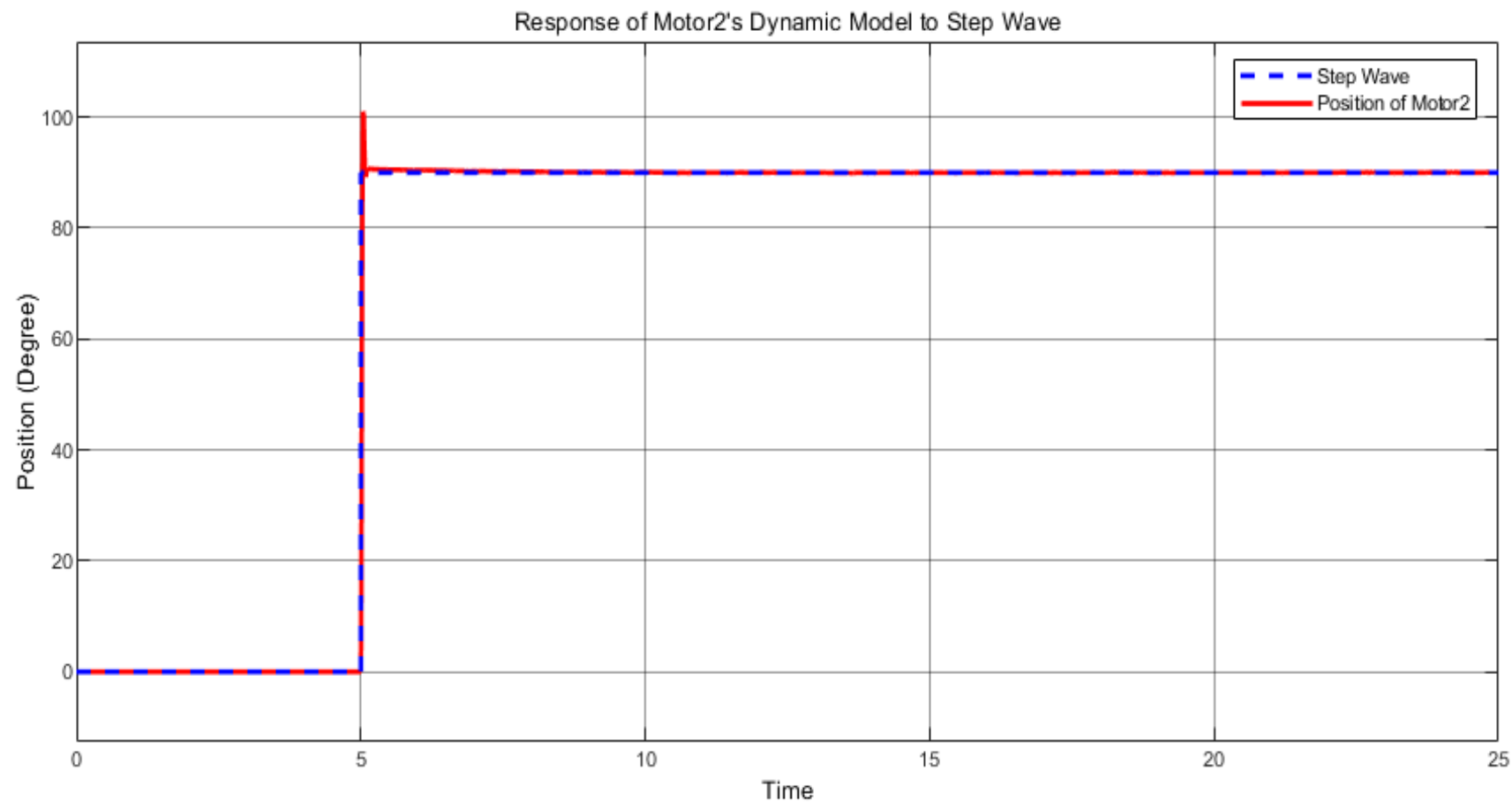

(b)

Figure 15. Joint 2 response a) sinus input, b) step input

As shown in Fig.16 the dynamic model for joint 3 is tracking the sine wave and step wave properly without delays and vibration in simulation. 


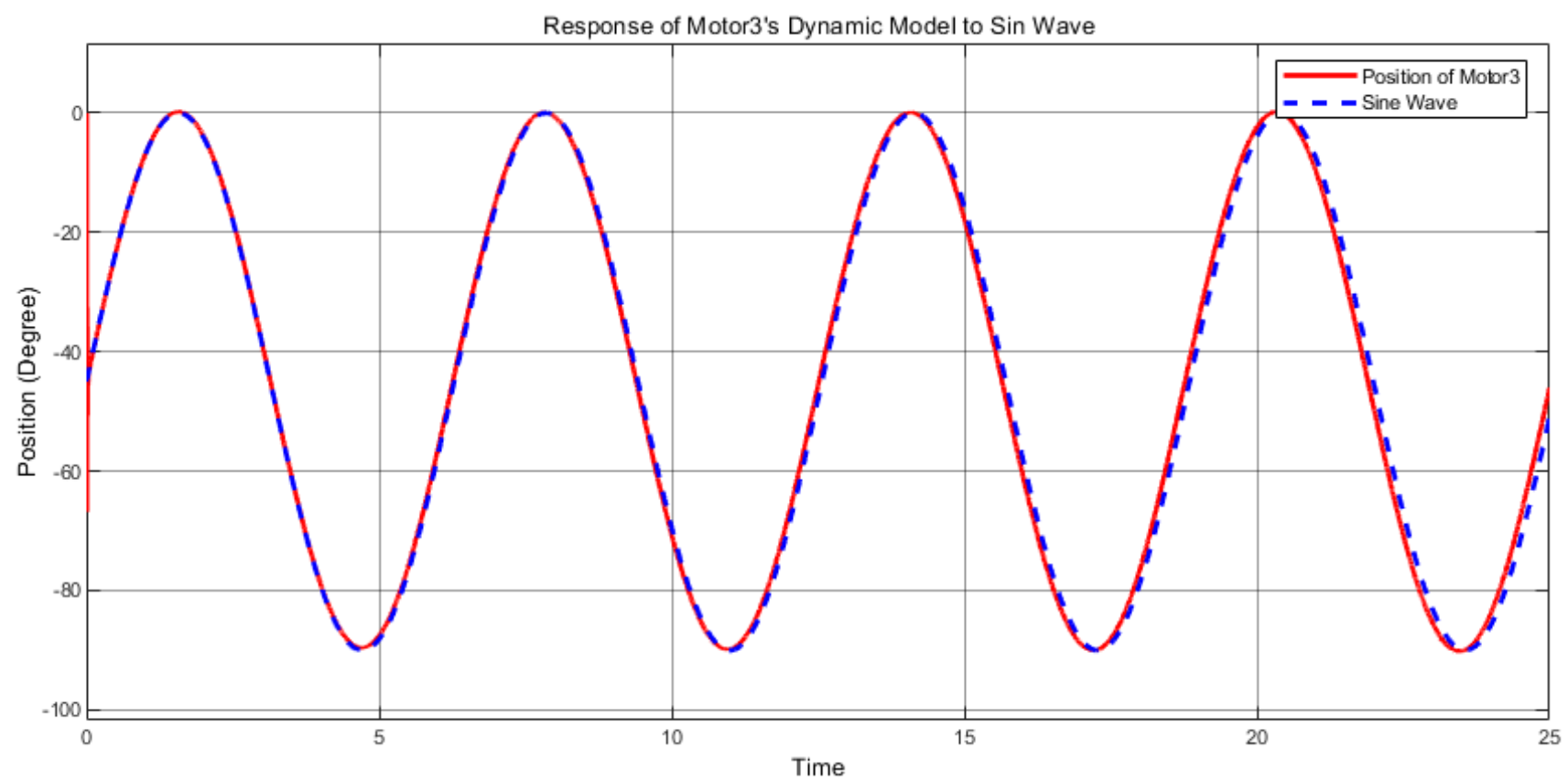

(a)

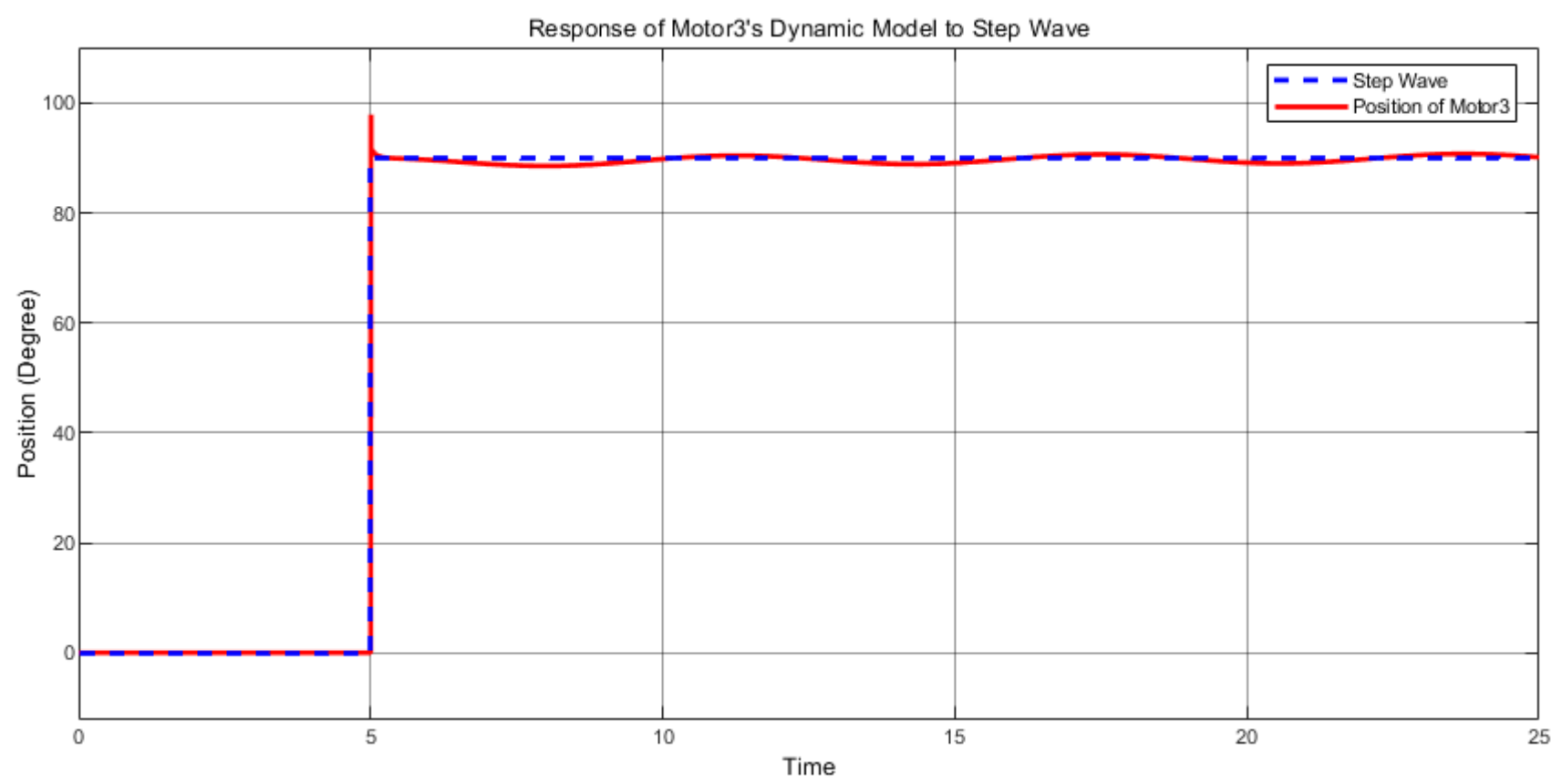

(b)

Figure 16. Joint 3 response a) sinus input, b) step input

\subsection{Simulation of passive exercise}

In the Simscape model, a trajectory was determined to require the use of all three motors in sequence. This trajectory was transferred to the system using switches and step functions. The switches are used to obtain a continuous trajectory tracking of all three movements. As can be seen in Fig. 17 the joint 1 can follow the reference step trajectory between 5-10 seconds. The joint 2 between 10-15 seconds and joint 3 between 20-25 seconds. The system works without delays and vibration.

All three motors give response in 5 seconds and follows the given trajectory without any overshoots. These results show that the PID constants were determined as correct. Therefore, this controller can be used for any desired trajectory. 


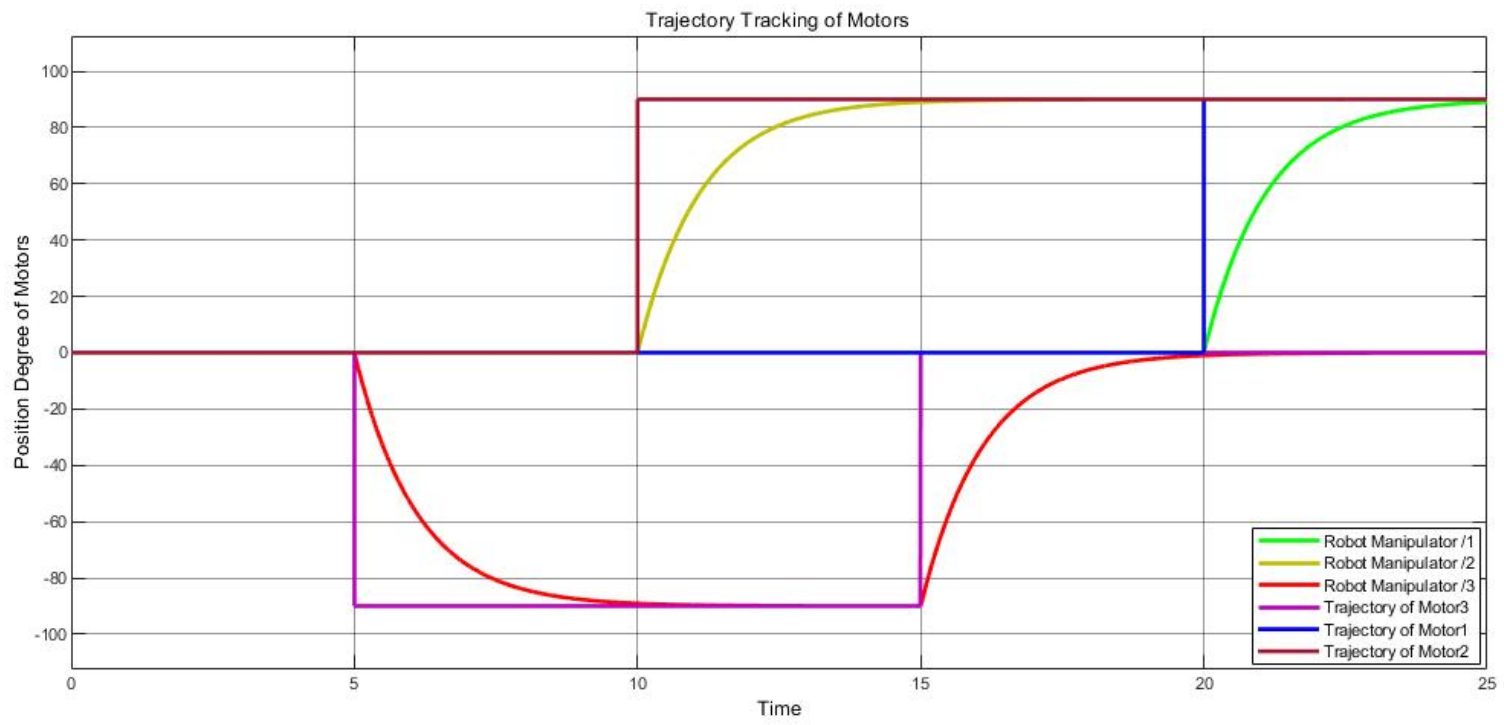

Figure 17. Trajectory of passive exercise

\subsection{Passive exercise with prototype}

This exercise can be applied on patients with no muscle activity in physical medicine and rehabilitation. The limbs of the patient move with any resistance. Physiotherapist can manage the exercise via graphical user interface. In order to generate desired trajectory, a Simulink real-time model is developed. In this system the encoder gets the position data from the robot as feedback. This data goes into the system as feedback and helps for positioning in desired position. PID controller controls the signal and gives the needed torque value into the subsystem which converts the torque value into voltage value to drive the motors properly after that the voltage value passes a saturation value for safety. Finally, after the last saturation the output signal exits the control system from the analog output port of the data acquisition card. To determine the PID parameters trial and error method is used. As a first step proportional gain $(P)$ is determined. The overshoot was considered during this determination. After that derivative gain $(D)$ is determined. Derivative value effected the response speed and noise sensitivity so these factors are considered during the process. Finally, the integral parameter $(I)$ is determined. This parameter is effective to decrease the steady-state error but as a disadvantage it can slower the response. With this methodology the PID parameters are selected as;

Joint 1: $P=50, I=35, D=5$

Joint 2: $P=20, I=0, D=5$

Joint 3: $P=70, I=37, D=5$

The responses for all three motors to a step function are given in Fig.18. As can been from the figures all three motors are working stable. All three motors have acceptable settling times which is around 3 seconds.

The results of the passive exercise experiment with different trajectories given in Fig. 19 for three joints. In Fig. 19a, a sinusoidal trajectory generated between $-45 \mathrm{deg}$ and $45 \mathrm{deg}$ and applied as desired input for joint 1 . It is clear that, robot manipulator is able to follow desired trajectory with high accuracy. For joint 2 , a sinusoidal trajectory generated between -30deg and 30deg and applied as desired input. Similar to Joint 1, this joint can also be followed the desired trajectory controller via the produces control signal generated by developed controller. Finally, a trapezoidal trajectory was generated and applied to joint 3. Also, desired trapezoidal trajectory can be followed by robot arm with very high accuracy. 


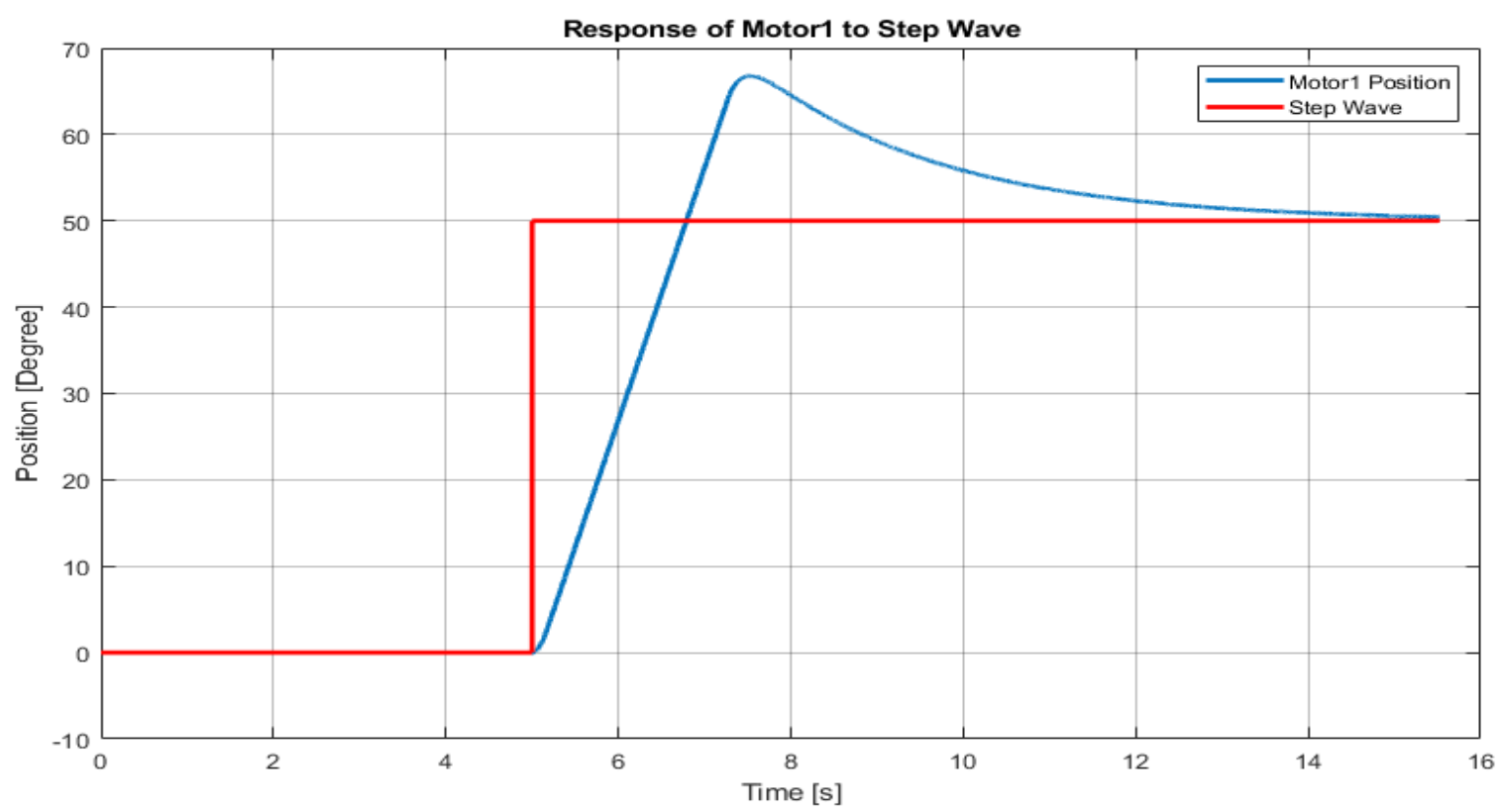

(a)

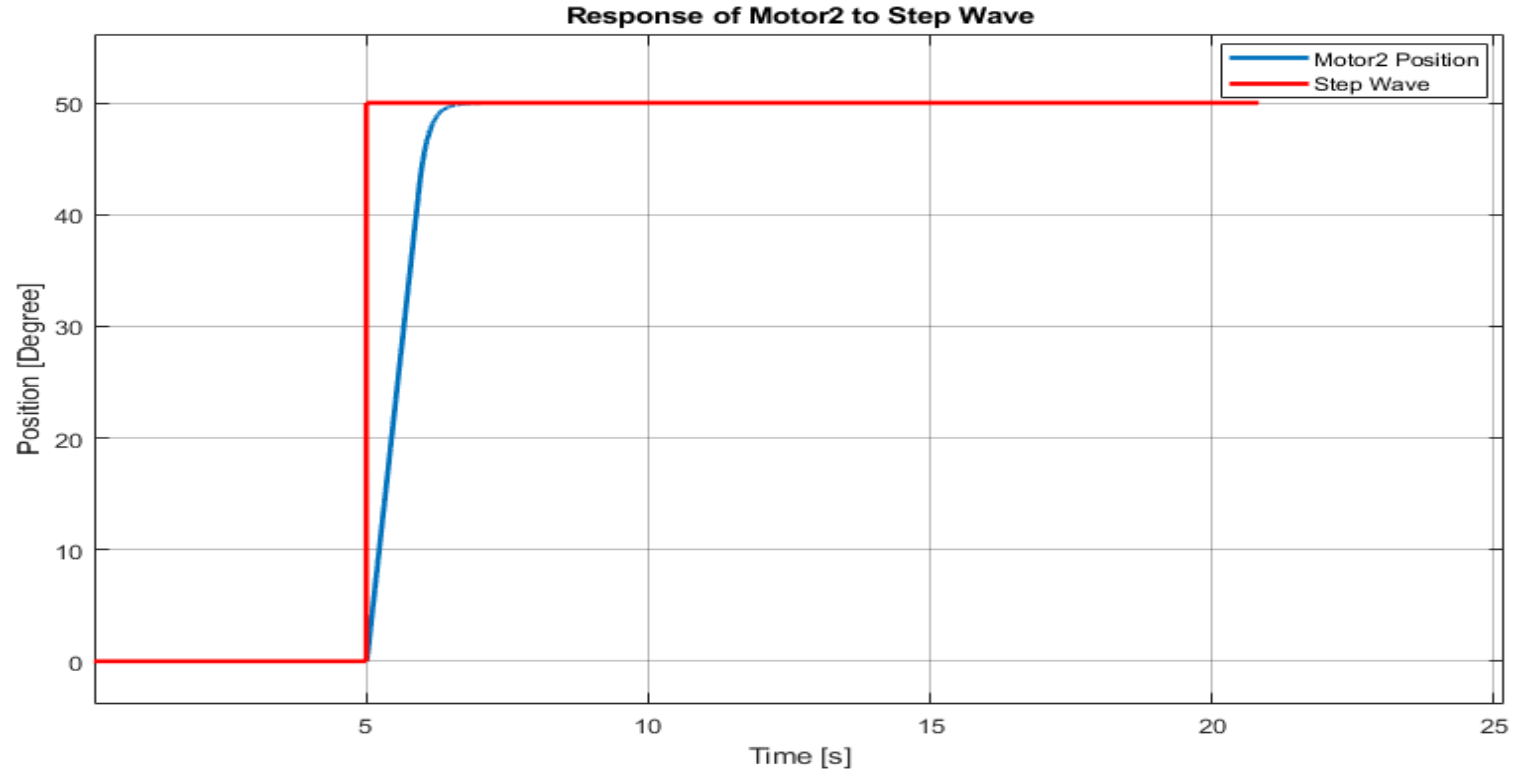

(b)

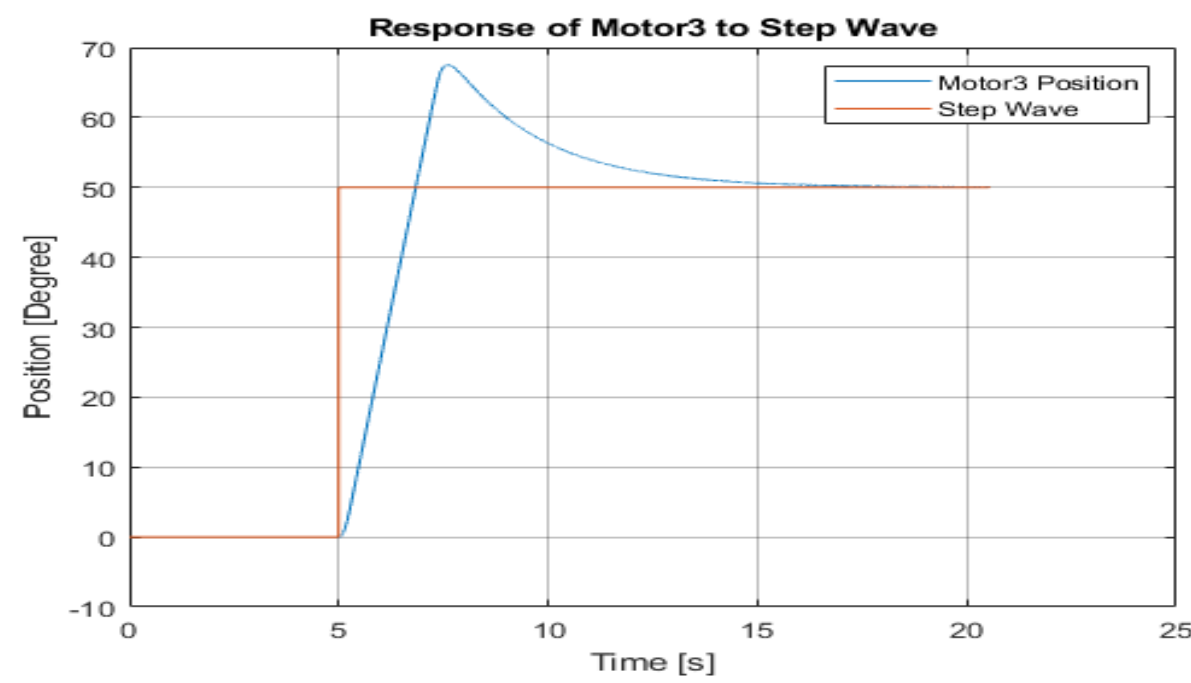

(c)

Figure 18. Step response of the system, a) Joint 1, b) Joint 2 c) Joint 3 


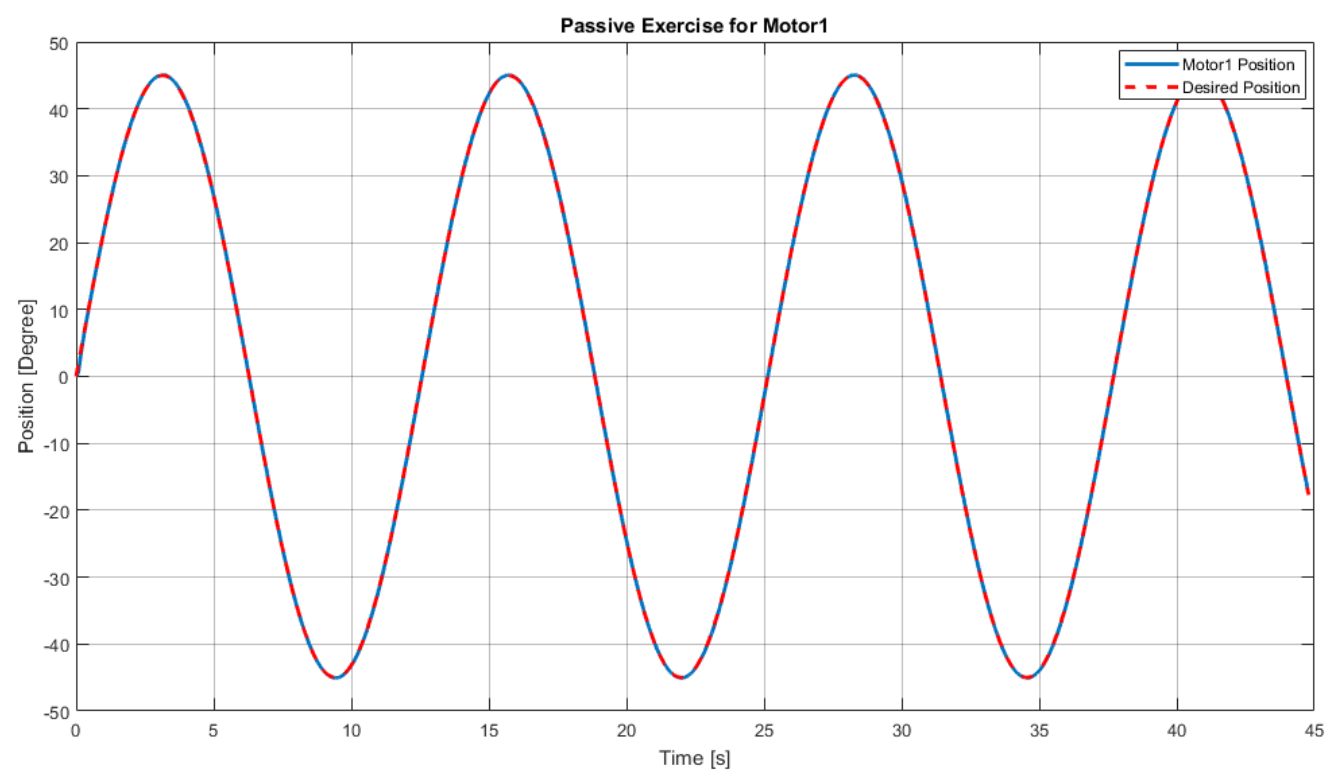

(a)

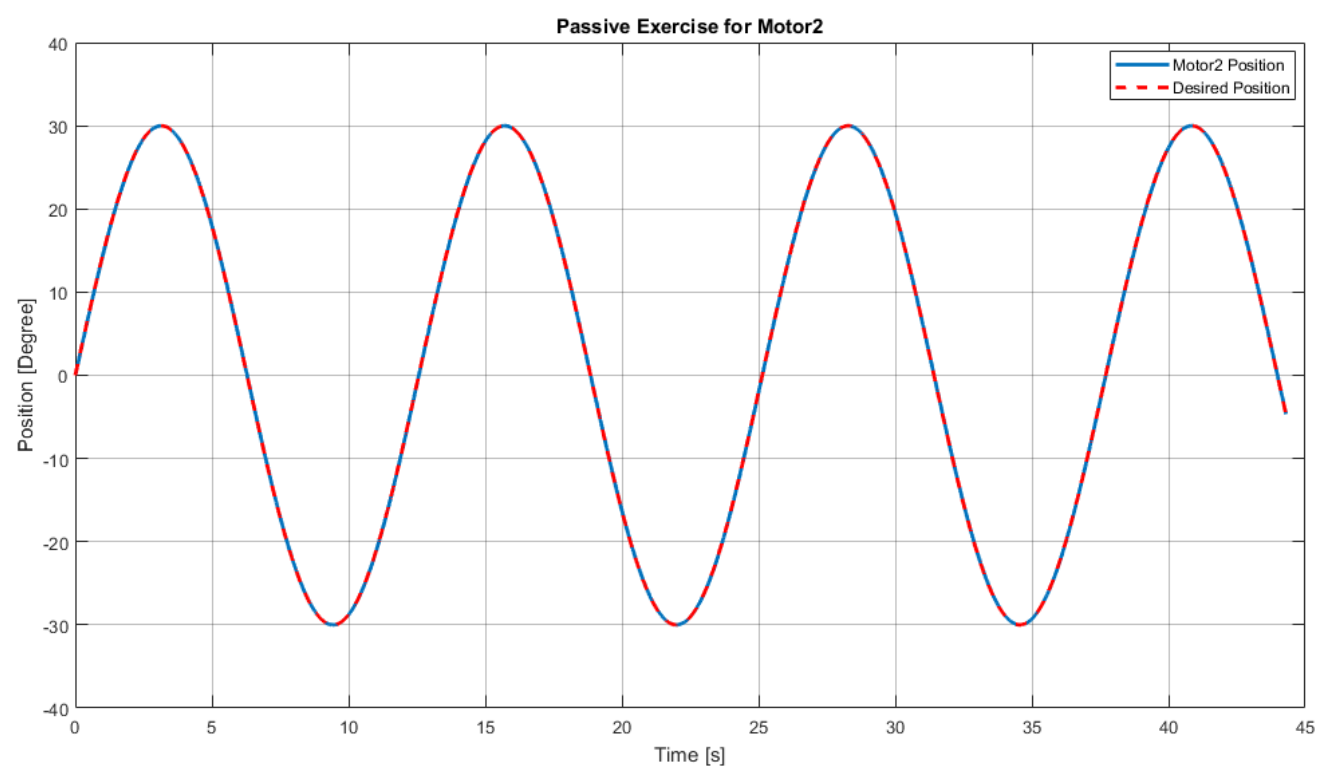

(b)

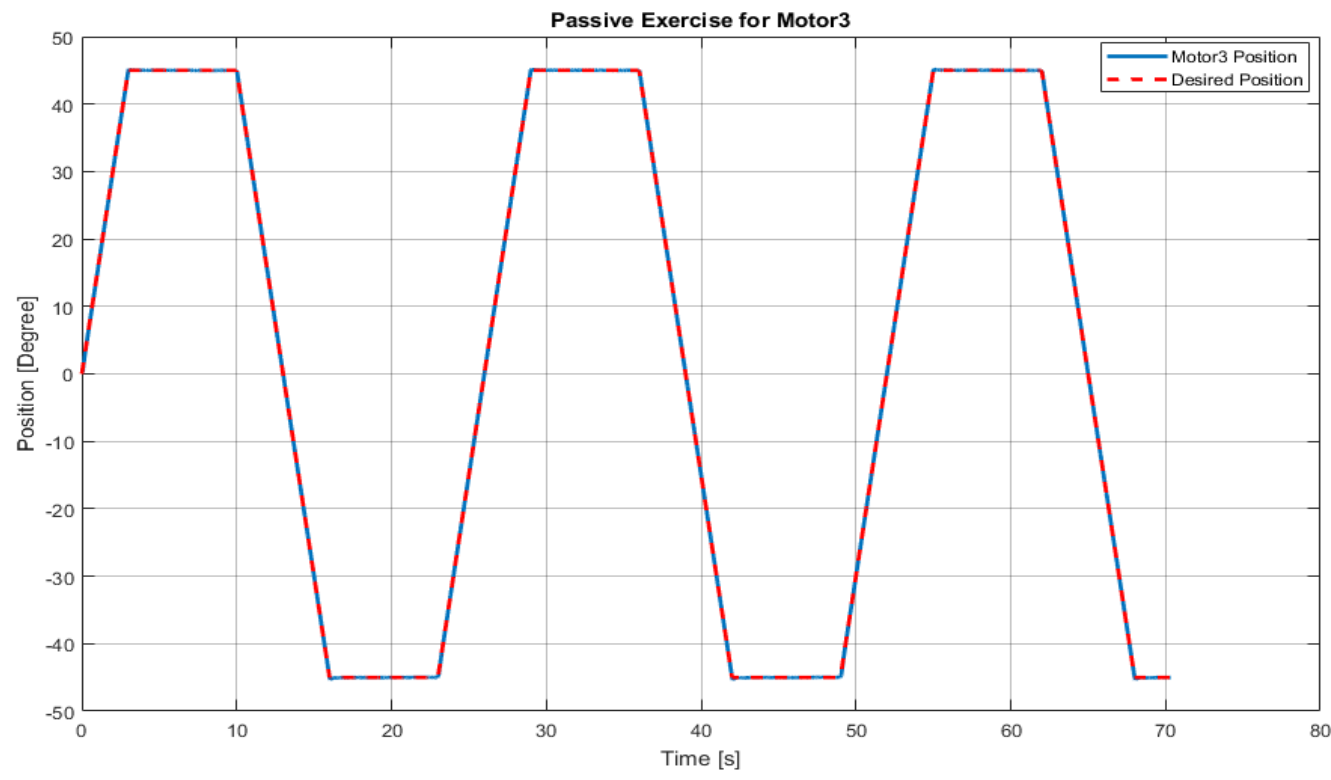

Figure 19. Passive Exercise Results (a) Joint 1, (b) Joint 2, (c) Joint 3 


\section{Conclusion}

In this study, a 4-DOF grounded exoskeletal robot for shoulder and elbow rehabilitation was designed, produced and control. The main purpose was to design a rehabilitation robot which has a basic structure and appropriate for home usage. Besides these it must also be cost effective. The robot can perform passive, active-assistive, isometric and isotonic exercises. The mechanical properties, system requirements, hardware, mathematical modelling were explained. A PID controller were developed for position-controlled exercises. Since the most known position-controlled exercise is passive exercise, simulation and test results with the prototype were given for passive exercise. According to test results, system can perform passive exercise with high accuracy.

The impedance controller will be developed for force-controlled exercises and the experimental results that are related to force-controlled exercises such as active-assistive, isometric and isotonic exercises will be presented in the next study.

\section{Acknowledgements}

This work was supported by the Scientific and Technological Research Council of Turkey (TUBITAK) under grant number 1139B411900912.

\section{References}

[1] E. Akdoğan, "Upper limb rehabilitation robot for physical therapy: design, control and testing," Turkish Journal of Electrical Engineering \& Computer Sciences, 24(3), 911-934, 2016.

[2] http://iknow.stpi.narl.org.tw/Post/Files/policy/2012/policy_12_037_2.pdf [Accessed, April 21, 2020]

[3] http://www.who.int/mediacentre/factsheets/fs317/en/ [Accessed, April 21, 2020]

[4] C.G. Burgar, P.S. Lum, P.C. Shor and H.F.M. Van der Loos, "Development of robots for rehabilitation therapy: The Palo Alto VA/Stanford experience, " Journal of Rehabilitation Research and Development 37, 663-673, 2000.

[5] A. Gupta and M. K. O'Malley, "Design of a haptic arm exoskeleton for training and rehabilitation," IEEE/ASME Transactions on Mechatronics, 11(3), 280-289, 2006.

[6] R. Vertechy, A. Frisoli, A. Dettori, M. Solazzi and M. Bergamasco, "Development of a new exoskeleton for upper limb rehabilitation," IEEE International Conference on Rehabilitation Robotics, 188-193, 2009.

[7] Y. Ren, H. S. Park and L. Q. Zhang, "Developing a whole-arm exoskeleton robot with hand opening and closing mechanism for upper limb stroke rehabilitation," IEEE International Conference on Rehabilitation Robotics, 761-765, 2009.

[8] Y. Ganesan, S. Gobee and V. Durairajah., "Development of an Upper Limb Exoskeleton for Rehabilitation with Feedback from EMG and IMU Sensor," Procedia Computer Science, 76, 53-59, 2015.

[9] T. Lenzi, S.M.M. De Rossi, N. Vitiello and M.C. Carrozza, "Intention-based EMG control for powered exoskeletons," IEEE Transactions Biomedical Engineering, 59(8), 2180-2190, 2012.

[10] K. Kiguchi, K. Iwami, M. Yasuda, K. Watanabe and T. Fukuda, "An exoskeletal robot for human shoulder joint motion assist," IEEE/ASME Transactions on Mechatronics, 8 (1), 125-135, 2003.

[11] K. Kiguchi, T. Tanaka and T. Fukuda, "Neuro-fuzzy control of a robotic exoskeleton with EMG signals," IEEE Transactions on Fuzzy Systems, 12 (4), 481-490, 2004. 
[12] H.C. Hsieh, D.F. Chen, L. Chien and C.C. Lan, "Design of a Parallel Actuated Exoskeleton for Adaptive and Safe Robotic Shoulder Rehabilitation," IEEE/ASME Trans. Mechatron., 22, 2034$2045,2017$.

[13] J. Hunt, H. Lee and P. Artemiadis, "A novel shoulder exoskeleton robot using parallel actuation and a passive slip interface," Journal of Mechanisms and Robotics, 9 (1), art. no. 011002, 2017.

[14] V.W. Oguntosin, Y. Mori, H. Kim, S.J. Nasuto, S. Kawamura and Y. Hayashi, "Design and Validation of Exoskeleton Actuated by Soft Modules toward Neurorehabilitation-Vision-Based Control for Precise Reaching Motion of Upper Limb," Frontiers in Neuroscience, 11, 352, 2017.

[15] Z. F. Shao, X. Tang and W. Yi, "Optimal design of a 3-DOF cable-driven upper arm exoskeleton," Advanced Mechanical Engineering, 6, 157096, 2014.

[16] Q. Wu, X. Wang and F. Du, "Development and analysis of a gravity-balanced exoskeleton for active rehabilitation training of upper limb," Journal of Mechanical Engineering Science, 230, 3777-3790, 2016.

[17] A. Accogli, L. Grazi, S. Crea, A. Panarese, J. Carpaneto, N. Vitiello and S. Micera, "EMG-based detection of user's intentions for human-machine shared control of an assistive upper-limb exoskeleton, " In Wearable Robotics: Challenges and Trends; Springer Berlin Germany, pp. 181$185,2017$.

[18] W. Xu, B. Chu, E. Rogers, "Iterative learning control for robotic-assisted upper limb stroke rehabilitation in the presence of muscle fatigue," Control Engineering Practice, 2014, vol. 31, p. 63-72.

[19] A. Riani, T. Madani, A. Beneallegue, K. Djouani," Adaptive integral terminal sliding mode control for upper-limb rehabilitation exoskeleton," Control Engineering Practice, 2018, vol. 75, p. 108117.

[20] L. Zhang, L. I. Jianfeng, S. U., Peng, et al. "Improvement of human-machine compatibility of upper-limb rehabilitation exoskeleton using passive joints," Robotics and Autonomous Systems, 2019, vol. 112, p. 22-31.

[21] M. A. Alawan, A. N. N. Al- Subeeh, O. J. M. Al-Furaiji. "Simulating an induction motor multioperating point speed control using PI controller with neural network. "Periodicals of Engineering and Natural Sciences, 2019, vol. 7, no 3, p. 1478-1485.

[22] B. M. Atiyah, S. H. Yadgar, M. G. K. Alabdullah," Comparison of MATLAB Simulink application with PLC application of real-time classical PID controllers in laboratory. "Periodicals of Engineering and Natural Sciences, 2019, vol. 7, no 4, p. 2045-2056.

[23] L. Zhang, G. Liu, B. Han, et al, "Assistive devices of human knee joint: A review." Robotics and Autonomous Systems, 2020, vol. 125, p. 103394.

[24] Y. Zhao, C. Liang, Z. Gu, Y. Zheng, Q. Wu, "A New Design Scheme for Intelligent Upper Limb Rehabilitation Training Robot," International Journal of Environmental Research and Public Health. 17. 2948. 10.3390/ijerph17082948, 2020.

[25] R.A.R.C. Gopura, D. S. V. Bandara, K. Kiguchi, G.K.I. Mann, "Developments in hardware systems of active upper-limb exoskeleton robots: A review," Robotics and Autonomous Systems, 2016, vol. 75 , p. 203-220.

[26] A. Rodic, S. Popic, M. Jovanovic, "Design and operation of exoskeletons for limb replacement or performance enhancement. In: Design and Operation of Human Locomotion Systems," Academic Press, 2020. p. 109-158.

[27] https://www.acefitness.org/ [Accessed, April 21, 2020] 
[28] Y. Rosales, R. López, S. Salazar, R. Lozano,” Design and modeling of an upper limb exoskeleton.” 266-272. 10.1109/ICSTCC.2015.7321304. (2015)

[29] E. Akdogan, M. A. Adli, "The design and control of a therapeutic exercise robot for lower limb rehabilitation: Physiotherabot," Mechatronics, vol.21, pp.509-522, 2011

[30] E. Akdoğan, E. Taçgın, M. Adli, A. "Knee rehabilitation using an intelligent robotic system," Journal of Intelligent Manufacturing, vol. 20, no. 2, pp. 195-202, 2009. 